\title{
BOUNDS AND APPROXIMATIONS FOR MULTISTAGE STOCHASTIC PROGRAMS*
}

\author{
FRANCESCA MAGGIONI ${ }^{\dagger}$ AND GEORG CH. PFLUG ${ }^{\ddagger}$
}

\begin{abstract}
Consider (typically large) multistage stochastic programs, which are defined on scenario trees as the basic data structure. It is well known that the computational complexity of the solution depends on the size of the tree, which itself increases typically exponentially fast with its height, i.e., the number of decision stages. For this reason approximations which replace the problem by a simpler one and allow bounding the optimal value are of great importance. In this paper we study several methods to obtain lower and upper bounds for multistage stochastic programs and we demonstrate their use in a multistage inventory problem.
\end{abstract}

Key words. multistage stochastic programming, bounds, refinement chain, expected value problem

AMS subject classifications. 90C15, 90C90,65K05

DOI. $10.1137 / 140971889$

1. Introduction. For very large multistage stochastic programs, the solution to optimality may be quite tedious and it often requires high-performance computers coupled with parallel solution algorithms.

Bounding techniques allow one to get quick information about lower and upper bounds for the objective value; if the gap between those two values is driven to an acceptably small value, the solution obtained may be considered good enough and a full optimization can be avoided altogether.

The main general principle for bounding minimization problems is the relaxation of some constraints to obtain lower bounds and the calculation of feasible solutions to get upper bounds. Many authors have found bounds following this strategy; see, e.g., [14], [13], [12]. Additional bounds can be found by methods of convex analysis (Jensen's inequality, Edmundson-Madansky inequality); see, e.g., [6], [7], [8], [9]. While the latter approaches exploit the approximation of continuous state and/or continuous time models by discretization, we assume in this paper that a discretized (large) tree model is already given and study alternative ways to find easy-to-compute bounds. These bounds are found by solving many much smaller problems instead of one big problem. The idea of looking at subproblems with only two scenarios goes back to [2] for the two-stage linear case (see also [3] and [18]). An extension to the multistage linear case is in [11].

In this paper we further extend the scope of the bounds calculation methodology by considering multistage convex problems with concave risk functionals as objective and by giving full sequences of bounds. We construct new refinement chains of lower bounds, where each bound can be computed by solving sets of group subproblems less complex than the original one, and recalculating the probabilities of each scenario in

\footnotetext{
*Received by the editors June 9, 2014; accepted for publication (in revised form) February 11, 2016; published electronically March 23, 2016.

http://www.siam.org/journals/siopt/26-1/97188.html

${ }^{\dagger}$ Department of Management, Economics and Quantitative Methods, University of Bergamo, Via dei Caniana 2, 24127 Bergamo, Italy (francesca.maggioni@unibg.it). This author was supported by the grant "Fondi di Ricerca di Ateneo di Bergamo 2013."

${ }^{\ddagger}$ Department of Statistics and Operations Research and International Institute for Applied Systems Analysis (IIASA), University of Vienna, Laxenburg, Austria (georg.pflug@univie.ac.at).
} 
the group accordingly. Refinement chains are constructed principally in two ways: by keeping one or several scenarios fixed in all subproblems or choosing them disjoint. A monotonically nondecreasing behavior in the cardinality of scenarios of each subproblem is proved.

Lower bounds for replacing the scenario process by its expectation are also considered. Several upper bounds obtained by inserting feasible solutions derived from smaller subproblems are presented generalizing the multistage expected value of the reference scenario (MEVRS) and the multistage expectation of pairs expected value (MEPEV) introduced in [11]. Relations among them are derived.

Notice that the proposed decomposition gets its justification by the fact that the complexity of a solution method for tree structured problems increases superlinearly with the problem size, sometimes even exponentially. Furthermore, the proposed approach has the advantage of splitting a given problem into independent subproblems so that parallel algorithms with high speed-up factors can easily be devised and used. Consequently, multistage problems, which are typically computationally complex and often not solvable by commercial solvers, could be handled by the proposed bounding technique. If it turns out that the calculation of lower bounds in the refinement chain is quick, then one may possibly find a good upper bound resulting in a solution gap which is small enough to stop the procedure here and not solve the big problem at all.

In the following, we assume that the stochastic program $O p t(\mathbb{P})$ is of the form ${ }^{1}$

$$
\operatorname{Opt}(\mathbb{P}): \quad v^{*}(\mathbb{P})=\min \left\{\mathcal{R}_{\mathbb{P}}[H(x, \xi)]: x \in \mathbb{X}, x \triangleleft \mathfrak{F} ; \mathbb{P} \sim(\Omega, \mathfrak{F}, P, \xi)\right\},
$$

where $\Omega$ is a finite but possibly large probability sample space, $\mathfrak{F}$ is a filtration on $\Omega$, i.e., an increasing sequence of sigma-algebras

$$
\mathfrak{F}=\left(\mathcal{F}_{0}=(\Omega, \emptyset), \mathcal{F}_{1}, \ldots, \mathcal{F}_{T}=2^{\Omega}\right),
$$

$\xi=\left(\xi_{0}, \ldots, \xi_{T}\right)$ is a stochastic process adapted to the filtration $\mathfrak{F}$ (in symbol $\xi \triangleleft \mathfrak{F}$ ), $P$ is the probability distribution on $\mathfrak{F}$, and $\mathbb{X}$ is the feasible set in which all decisions must lie, i.e., $x(\omega) \in \mathbb{X}$ for all $\omega \in \Omega$. The structure $(\Omega, \mathfrak{F}, P, \xi)$ is summarized by the symbol $\mathbb{P}^{2} H(x, \xi)$ is the cost function and $\mathcal{R}$ is a risk functional, like the expectation or the average value-at-risk.

The finite probability space $\Omega$ endowed with the filtration $\mathfrak{F}$ of length $T+1$ as in (1.2) defines a finite tree of height $T$ with leaf nodes $\Omega$. The probability $P$ defined on $\Omega$ induces probabilities on all nodes $\mathfrak{N}$ of the tree. The distance of every node from the root is its stage. The nodes at stage $t$ are denoted by $\mathfrak{N}_{t}$, where $\mathfrak{N}_{0}$ is a singleton (the root) and $\mathfrak{N}_{T}=\Omega$ (the leaves). The stochastic scenario process $\xi$ can be seen as defined on the node set $\mathfrak{N}$.

We exploit the special structure of the problem $O p t(\mathbb{P})$. For a simpler presentation we often omit some components of the model $\mathbb{P} \sim(\Omega, \mathfrak{F}, P, \xi)$ and write, for instance, just $\mathbb{P} \sim(\mathfrak{F}, P)$ if both the probability space $\Omega$ and the random process $\xi$ are fixed and no confusion is possible.

The paper is organized as follows. Lower bounds are introduced in sections 2 and 3 by dissecting the probability measure and filtration and by replacing the scenario

\footnotetext{
${ }^{1}$ We assume throughout the paper that all optimal values are attained so that we write min and not inf.

$2 \mathbb{P}$ describes the equivalence class of all models which can be bijectively mapped to the structure $(\Omega, \mathfrak{F}, P, \xi)$. This equivalence class is called the nested distribution; see [16]. However, for reading this paper one may always assume that a concrete model $(\Omega, \mathfrak{F}, P, \xi)$ is given.
} 
process by its expectation respectively. Upper bounds by inserting suboptimal solutions are analyzed in section 4 . Lower and upper bounds introduced in the previous sections are then tested on a multistage inventory problem in section 5. Section 6 deals with complexity considerations and section 7 concludes the paper.

2. Lower bounds by dissecting the probability measure. Given the optimal value mapping $(\mathfrak{F}, P, \xi) \sim \mathbb{P} \longmapsto v^{*}(\mathbb{P})=v^{*}(\mathfrak{F}, P, \xi)$ we keep the filtration and the process $\xi$ fixed and consider only the mapping

$$
P \mapsto v^{*}(P) .
$$

One main structural property of some stochastic programs (including those of the expectation type) is that this mapping is concave.

Lemma 2.1. Suppose that in the basic problem (1.1) the functional $P \longmapsto \mathcal{R}_{P}(\cdot)$ is concave (i.e., the mapping $P \mapsto \mathcal{R}_{P}(Y)$ is concave for all random variables $Y$ for which $\mathcal{R}$ is defined; see [15]). Then the mapping $P \mapsto v^{*}(P)$ is also concave.

Proof. Let $P=\sum_{i=1}^{k} p_{i} P_{i}$ with $\sum p_{i}=1, p_{i}>0$, and let $x^{*}$ be a solution of problem $\operatorname{Opt}(P)=\operatorname{Opt}(\mathfrak{F}, P, \xi)$. Since $x^{*}$ is feasible for all $\operatorname{Opt}\left(P_{i}\right)$ we have that

$$
v^{*}(P)=\mathcal{R}_{P}\left[H\left(x^{*}, \xi\right)\right] \geq \sum p_{i} \mathcal{R}_{P_{i}}\left[H\left(x^{*}, \xi\right)\right] \geq \sum p_{i} v^{*}\left(P_{i}\right) .
$$

Notice that the lemma holds for any feasible set $\mathbb{X}$. In particular it holds for integer or mixed integer problems.

Numerous consequences result from this simple Lemma 2.1. For instance, let $\mathfrak{N}_{T}=\Omega=\left\{\omega_{1}, \ldots, \omega_{k}\right\}$ be the leaf set of the finite tree. Let $\delta_{\omega_{i}}$ be the point mass at scenario $\omega_{i}$. Under $\delta_{\omega_{i}}$ the scenario $\omega_{i}$ has probability 1 and all other scenarios have probability zero. Thus solving the problem under $\delta_{\omega_{i}}$ leads to a deterministic dynamic optimization problem, with optimal value denoted by $v^{*}\left(\delta_{\omega_{i}}\right)$. Notice that

$$
P=\sum_{i=1}^{k} P\left(\omega_{i}\right) \delta_{\omega_{i}} .
$$

The value $v_{1}^{*}:=\sum_{i} P\left(\omega_{i}\right) v^{*}\left(\delta_{\omega_{i}}\right)$ is the clairvoyant's solution, which may alternatively be written as

$$
v_{1}^{*}=v^{*}\left(\mathfrak{F}^{T}, P\right),
$$

where $\mathfrak{F}^{T}$ is the clairvoyant's filtration $\mathfrak{F}^{T}=\left(\mathcal{F}_{T}, \ldots, \mathcal{F}_{T}\right)$. By (2.1) and Lemma 2.1 we get the (quite trivial) lower bound by the inequality

$$
v_{1}^{*}=v^{*}\left(\mathfrak{F}^{T}, P\right) \leq v^{*}(\mathfrak{F}, P) .
$$

Some authors would call this inequality

$$
\text { wait-and-see } \leq \text { here-and-now }
$$

which is a bit misleading, since the essence of the "wait-and-see" solution is here not to wait but to decide immediately with complete knowledge of the future, an ability which we may attribute only to clairvoyants.

Remark. The inequality

$$
v^{*}(\mathfrak{F}, P) \geq v^{*}\left(\mathfrak{F}^{T}, P\right)
$$


is just one example of the fundamental but quite trivial inequality

$$
\mathfrak{F}_{1} \subseteq \mathfrak{F}_{2} \quad \text { implies that } \quad v^{*}\left(\mathfrak{F}_{1}, P\right) \geq v^{*}\left(\mathfrak{F}_{2}, P\right) .
$$

This inequality can produce lower and upper bounds: by refining the filtration, i.e., relaxing the nonanticipativity constraints, one gets lower bounds, and by coarsening it, one gets upper bounds.

Refinement chains. While the dissection of $P$ into its atoms $\left(\delta_{\omega_{i}}\right)$ is the most extreme dissection, one may also dissect it into a convex combination of probabilities defined on two or more (but not all) leaves. For instance, let $\omega_{1}$ be a specific leaf node (without loss of generality (w.l.o.g.) call it $\omega_{1}$ ) and set for $i \neq 1$

$$
P_{i}^{(2)}=P\left(\omega_{1}\right) \delta_{\omega_{1}}+\left(1-P\left(\omega_{1}\right)\right) \delta_{\omega_{i}} .
$$

Defining $\pi_{i}^{(2)}=P\left(\omega_{i}\right) /\left(1-P\left(\omega_{1}\right)\right)$ one sees that $P=\sum_{i \neq 1} \pi_{i}^{(2)} P_{i}^{(2)}$ and hence

$$
v_{2}^{*}:=\sum_{i \neq 1} \pi_{i}^{(2)} v^{*}\left(P_{i}^{(2)}\right) \leq v^{*}(P) .
$$

Thus a lower bound for the basic problem can be found by solving $k-1$ multistage problems with only two scenarios each. Since by (2.4) every subproblem with two scenarios is a convex combination of two (clairvoyant's) problems with just one scenario each, one gets for $i \neq 1$

$$
v^{*}\left(P_{i}^{(2)}\right) \geq P\left(\omega_{1}\right) v^{*}\left(\delta_{\omega_{1}}\right)+\left(1-P\left(\omega_{1}\right)\right) v^{*}\left(\delta_{\omega_{i}}\right),
$$

and one arrives at a chain of inequalities

$$
v_{1}^{*}=\sum_{i=1}^{k} P\left(\omega_{i}\right) v^{*}\left(\delta_{\omega_{i}}\right) \leq v_{2}^{*} \leq v^{*}(P) .
$$

Lemma 2.2. If $\Omega=\left\{\omega_{2}, \ldots, \omega_{k}\right\}$ and $\omega_{1} \notin \Omega$, then $v_{1}^{*}=v_{2}^{*}$.

Proof. See [11] for the proof.

Pairs of scenarios were considered for the first time in [2], in [3] and [18] for the two-stage linear case, and in [11] for the multistage linear case by means of the definition of multistage sum of pairs expected values. Inequality (2.5) has been proven in [11] for the multistage linear case with risk functional being the expectation.

The method may be refined by considering longer refinement chains. Such a chain has the structure

$$
\begin{gathered}
\Omega \\
\vdots \\
\left(\Omega_{1}^{(j)}, \Omega_{2}^{(j)}, \ldots, \Omega_{m_{j}}^{(j)}\right) \\
\vdots \\
\left(\Omega_{1}^{(2)}, \Omega_{2}^{(2)}, \ldots, \Omega_{m_{2}}^{(2)}\right) \\
\left(\left\{\omega_{1}\right\},\left\{\omega_{2}\right\}, \ldots,\left\{\omega_{k}\right\}\right),
\end{gathered}
$$


where each row is a collection of subsets of the probability space $\Omega$ with the property that their union covers the whole space $\Omega=\cup_{i} \Omega_{i}^{(j)}$ for all $j$ and that each set $\Omega_{i}^{(j)}$ is the union of sets from the next more refined collection,

$$
\Omega_{i}^{(j)}=\cup_{\Omega_{s}^{(j-1)} \subseteq \Omega_{i}^{(j)}} \Omega_{s}^{(j-1)} .
$$

For each refinement chain of the probability space $\Omega$ there is a correspondent chain of dissections of the probability $P$ into probability measures $P_{i}^{(j)}$,

$$
\begin{gathered}
P \\
\vdots \\
\left(P_{1}^{(j)}, \ldots P_{m_{j}}^{(j)}\right) \\
\vdots \\
\left(P_{1}^{(2)}, \ldots, P_{m_{2}}^{(2)}\right) \\
\left(P_{1}^{(1)}=\delta_{\omega_{1}}, \ldots, P_{k}^{(1)}=\delta_{\omega_{k}}\right)
\end{gathered}
$$

such that

(i) $P_{i}^{(j)}$ has support $\Omega_{i}^{(j)}$,

(ii) $P$ can be written as $P=\sum_{i=1}^{m_{j}} \pi_{i}^{(j)} P_{i}^{(j)}$,

(iii) each $P_{i}^{(j)}$ can be written as a convex combination of probabilities from the refined collection $\left\{P_{i}^{(j-1)}\right\}$.

It is evident that such a refinement chain leads to a chain of lower bounds. Denoting

$$
v_{j}^{*}=\sum_{i=1}^{m_{j}} \pi_{i}^{(j)} v^{*}\left(P_{i}^{(j)}\right),
$$

by means of Lemma 2.1 as well as (ii) and (iii) we get a chain of lower bounds expressed as follows:

$$
v_{1}^{*} \leq v_{2}^{*} \leq \cdots \leq v_{j}^{*} \leq \cdots \leq v^{*}(P) .
$$

Notice that the higher the index $j$, the fewer the problems that have to be solved, but with an increasing number of scenarios. Notice also that, as expected, the clairvoyant solution always gives the smallest lower bound in this chain.

There are many ways to construct refinement chains. One may keep one or several scenarios fixed in all subsets $\Omega_{i}^{(j)}$ or choose them disjoint. Two examples will illustrate some possible choices.

Example 2.3. One fixed scenario. Suppose that scenario $\omega_{1}$ is fixed and appears in all subsets. Then the structure of the refinement chain is

$$
\begin{gathered}
\left(\left\{\omega_{1}, \omega_{2}, \ldots, \omega_{k}\right\}\right) \\
\left(\left\{\omega_{1}, \omega_{2}, \ldots, \omega_{s}\right\},\left\{\omega_{1}, \omega_{s+1}, \ldots, \omega_{k}\right\}\right) \\
\vdots \\
\left(\left\{\omega_{1}, \omega_{2}\right\},\left\{\omega_{1}, \omega_{3}\right\}, \ldots,\left\{\omega_{1}, \omega_{k}\right\}\right) .
\end{gathered}
$$



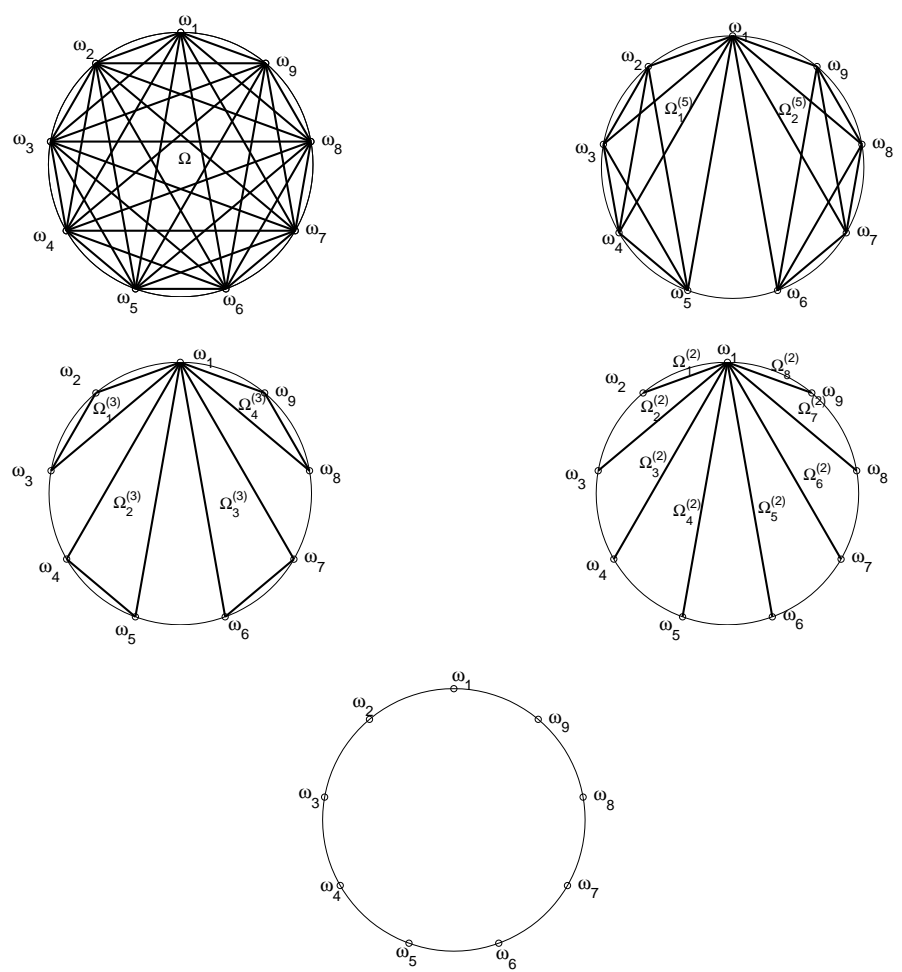

FIG. 2.1. A graphical representation of a refinement chain where only the scenario $\omega_{1}$ is fixed. The collection $\left(\Omega_{1}^{(2)}, \ldots, \Omega_{8}^{(2)}\right)$ is the "pair of scenarios" collection (with $\omega_{1}$ fixed). Similarly the collection $\left(\Omega_{1}^{(3)}, \ldots, \Omega_{4}^{(3)}\right)$ is the "triplet of scenarios" collection (with $\omega_{1}$ fixed) and $\left(\Omega_{1}^{(5)}, \Omega_{2}^{(5)}\right)$ for "quintuplets of scenarios."

The situation is illustrated in Figure 2.1. All scenarios which belong to a subset $\Omega_{i}^{(j)}$ are connected by edges and form a connected subgraph.

As a generalization, we consider the case where two or more scenarios may be fixed, i.e., they appear in all subsets. In order to calculate the correct weights $\pi_{i}^{(\cdot)}$ of the dissections, assume w.l.o.g. that the first $f$ scenarios $\left\{\omega_{1}, \ldots, \omega_{f}\right\}$ are fixed and appear in all subsets. Assume further that each subset contains exactly $j>f$ scenarios, meaning that the total number $s$ of subsets of cardinality $j$, i.e., $s=$ $(k-f) /(j-f)$, is an integer. Let $x=j-f>0$. The corresponding $s$ elements of the refinement chain are

$$
\Omega_{1}^{(\cdot)}=\left\{\omega_{1}, \ldots, \omega_{f}, \omega_{f+1}, \ldots, \omega_{f+x}\right\}, \Omega_{2}^{(\cdot)}=\left\{\omega_{1}, \ldots, \omega_{f}, \omega_{f+x+1}, \ldots, \omega_{f+2 x}\right\}, \ldots
$$

or in general

$$
\Omega_{i}^{(\cdot)}=\left\{\omega_{1}, \ldots, \omega_{f}, \omega_{f+(i-1) \cdot x+1}, \ldots, \omega_{f+i \cdot x}\right\}, \quad i=1, \ldots, s .
$$

The probabilities $P_{i}^{(\cdot)}$ as well as the corresponding weights $\pi_{i}^{(\cdot)}$ can be calculated as follows:

$$
P_{i}^{(\cdot)}=\sum_{m=1}^{f} P\left(\omega_{m}\right) \cdot \delta_{\omega_{m}}+\frac{\left(1-\sum_{m=1}^{f} P\left(\omega_{m}\right)\right)}{\sum_{m=f+(i-1) \cdot x+1}^{f+i \cdot x} P\left(\omega_{m}\right)} \sum_{m=f+(i-1) \cdot x+1}^{f+i \cdot x} P\left(\omega_{m}\right) \cdot \delta_{\omega_{m}}
$$



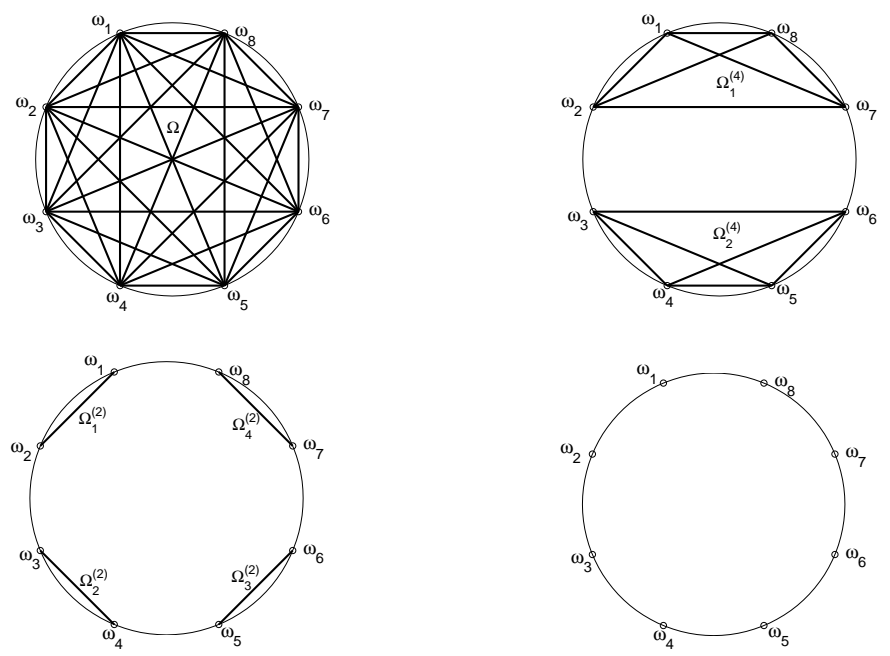

FIG. 2.2. A refinement chain with disjoint subsets.

and

$$
\pi_{i}^{(\cdot)}=\frac{\sum_{m=f+(i-1) \cdot x}^{f+i \cdot x} P\left(\omega_{m}\right)}{\left(1-\sum_{m=1}^{f} P\left(\omega_{m}\right)\right)}, \quad i=1, \ldots, s .
$$

With this choice one gets $\sum_{m=1}^{s} \pi_{i}^{(\cdot)}=1$ and $P=\sum_{i=1}^{s} \pi_{i}^{(\cdot)} P_{i}^{(\cdot)}$ and therefore the corresponding lower bound is

$$
\sum_{i=1}^{s} \pi_{i}^{(\cdot)} v^{*}\left(P_{i}^{(\cdot)}\right) \leq v^{*}(P)
$$

Notice that (2.5) is a special case with $f=1$ and $j=2$.

Example 2.4. Alternatively one may also consider disjoint partitions. In this case, the refinement chain corresponds to a filtration and the probability measures $P_{i}^{(j)}$ are

$$
P_{i}^{(j)}= \begin{cases}\frac{1}{\pi_{i}^{(j)}} P\left(\omega_{s}\right) \delta_{\omega_{s}} & \text { if } \omega_{s} \in \Omega_{i}^{(j)}, \\ 0 & \text { otherwise }\end{cases}
$$

with

$$
\pi_{i}^{(j)}=\sum_{\omega_{s} \in \Omega_{i}^{(j)}} P\left(\omega_{s}\right) .
$$

Figure 2.2 illustrates such a refinement chain.

3. Lower bounds for replacing the scenario process by its expectation. A popular choice for simplifying a dynamic stochastic problem is to replace the scenario process $\left(\xi_{t}\right)_{t=1, \ldots, T}$ by its expectation $\left(\mathbb{E}\left[\xi_{t}\right]\right)_{t=1, \ldots, T}$, if it exists. The basic problem (1.1) is modified to

$$
\operatorname{Opt}\left(\mathbb{E}_{P}\right): \quad \min \left\{\mathcal{R}\left[H\left(x, \mathbb{E}_{P} \xi\right)\right]: x \in \mathbb{X} ; x \triangleleft \mathfrak{F} ; \mathbb{P}=(\mathfrak{F}, P, \xi)\right\} .
$$

We call problem (3.1) the expectation-reduced problem. Notice that we have replaced the stochastic scenario process by a deterministic one but have kept the possibility 
for the decisions to depend on the filtration. One might argue that such random decisions for nonrandom scenarios is not appropriate. A closer look reveals in fact that the optimal decision for deterministic compound concave problems can be chosen as deterministic as well, i.e., it is not necessary to consider randomized decisions.

TheOREM 3.1. Suppose that the risk functional $\mathcal{R}_{P}$ is concave ${ }^{3}$ in $P$. Then the solution of the expectation-reduced problem (3.1) can be found for a deterministic decision sequence $\left(x_{0}^{*}, \ldots, x_{T-1}^{*}\right)$.

Proof. Notice that the expectation-reduced problem has a deterministic objective $\bar{H}(x)=H(x, \mathbb{E} \xi)$. Dissect the probability $P$ into the atoms defined on the leaves $P=$ $\sum_{i=1}^{k} P\left(\omega_{i}\right) \delta_{\omega_{i}}$. Then, by concavity, for any $x, \mathcal{R}_{P}[\bar{H}(x)] \geq \sum_{i=1}^{k} P\left(\omega_{i}\right) \mathcal{R}_{\delta_{\omega_{i}}}[\bar{H}(x)]$. Thus the optimal decisions can be found by solving the clairvoyant problems. But since all clairvoyant problems are equal, the same solution may be inserted for all scenarios, i.e., the solution may be chosen deterministic as well.

Let $\mathbb{P}_{\mathbb{E}(\xi)}$ be the degenerated (nested) distribution, which assigns probability one to the sequence $\mathbb{E} \xi_{1}, \ldots \mathbb{E} \xi_{T}$. Under the assumption of Theorem 3.1, one may w.l.o.g. assume that the solution of the deterministic problem

$$
v^{*}\left(\mathbb{P}_{\mathbb{E}(\xi)}\right)=\min \left\{\mathcal{R}_{\mathbb{P}_{\mathbb{E}(\xi)}}[H(x, \xi)]: x \in \mathbb{X}\right\}=\min \left\{\mathcal{R}\left[H\left(x, \mathbb{E}_{P} \xi\right)\right]: x \in \mathbb{X}\right\}
$$

is deterministic.

The value $v^{*}\left(\mathbb{P}_{\mathbb{E}(\xi)}\right)$ in the linear case is known in literature as expected value (see, for instance, [2] and many others).

DEFINITION 3.2. The random variable $X_{1}$ is dominated by the random variable $X_{2}$ in the second-order sense and we write $X_{1} \prec_{S S D} X_{2}$, where SSD stands for second-order stochastic dominance $[4]$, if $\mathbb{E}\left[U\left(X_{1}\right)\right] \leq \mathbb{E}\left[U\left(X_{2}\right)\right]$ for all monotonically increasing and concave utility functions $U$.

Lemma 3.3. Suppose that (i) $(x, \xi) \mapsto H(x, \xi)$ is convex, (ii) $P \mapsto \mathcal{R}_{P}$ is concave, and (iii) $\mathcal{R}=\mathcal{R}_{P}$ has the following property:

$$
-Y_{1} \prec_{S S D}-Y_{2} \quad \text { implies that } \quad \mathcal{R}\left(Y_{1}\right) \geq \mathcal{R}\left(Y_{2}\right) .
$$

Then, assuming that the solution of the basic problem (1.1) is integrable and that the set of feasible solutions $\mathbb{X}$ is convex,

$$
v^{*}\left(\mathbb{P}_{\mathbb{E}(\xi)}\right) \leq v^{*}(\mathbb{P}) .
$$

Proof. Suppose that $\eta=x^{*}(\xi)$ is the solution of (1.1). For all monotonically increasing and concave utility functions $U$, the function $V(y)=-U(-y)$ is convex and monotonically increasing. Since $(\eta, \xi) \mapsto V(H(\eta, \xi))$ is convex, by Jensen's inequality $\mathbb{E}\left[V(H(\eta, \xi)] \geq V(H(\mathbb{E}(\eta), \mathbb{E} \xi))\right.$. Let $x^{+}=\mathbb{E}(\eta)=\mathbb{E}\left(x^{*}(\xi)\right)$ (a deterministic function); then

$$
\mathbb{E}\left[U\left(-H\left(x^{*}(\xi), \xi\right)\right] \leq U\left(-H\left(x^{+}, \mathbb{E} \xi\right)\right)\right.
$$

and therefore by assumption $\mathcal{R}\left[H\left(x^{*}(\xi), \xi\right)\right] \geq \mathcal{R}\left[H\left(x^{+}, \mathbb{E} \xi\right)\right]$. By Theorem 1 , the solution of the expectation-reduced can be found among deterministic functions and therefore

$$
v^{*}(\mathbb{P})=\mathcal{R}\left[H\left(x^{*}(\xi), \xi\right)\right] \geq \mathcal{R}\left[H\left(x^{+}, \mathbb{E} \xi\right)\right] \geq v^{*}\left(\mathbb{P}_{\mathbb{E}(\xi)}\right),
$$

which is the asserted relation.

\footnotetext{
${ }^{3}$ If the functional is expected disutility, i.e., $\mathcal{R}(Y)=\mathbb{E}[V(Y)]$, then it is affine linear and a fortiori concave in $P$.
} 
Remark. Notice that the functional $\mathcal{R}(Y)=\mathbb{E}[V(Y)]$ with a monotonically increasing and convex function $V$ satisfies the conditions of the lemma, since $y \mapsto$ $-V(-y)$ is monotonically increasing and concave.

Example 3.4. The convexity of $(x, \xi) \mapsto H(x, \xi)$ is, for instance, ensured in the following decision model with random costs $c_{t}$ and random right-hand side $h_{t}$ in the linear constraints. Suppose that the problem is formulated as

$$
\begin{array}{r}
\min \left\{c_{0}\left(x_{0}\right)+\mathbb{E}_{\xi_{1}}\left[\min c_{1}\left(x_{1}, \xi_{1}\right)+\mathbb{E}_{\xi_{2}}\left[\min c_{2}\left(x_{2}, \xi_{2}\right)\right.\right.\right. \\
\left.\left.\left.+\mathbb{E}_{\xi_{T}}\left[\cdots+\mathbb{E}\left[\min c_{T}\left(x_{T}, \xi_{T}\right)\right] \ldots\right]\right]\right]: x \in \mathbb{X}\right\}
\end{array}
$$

where the feasible set $\mathbb{X}$ is given by

$$
\begin{aligned}
W_{0} x_{0} & \geq h_{0} \\
A_{1} x_{0}+W_{1} x_{1} & \geq h_{1}\left(\xi_{1}\right) \\
A_{2} x_{1}+W_{2} x_{2} & \geq h_{2}\left(\xi_{2}\right) \\
\vdots & \\
A_{T} x_{T-1}+W_{T} x_{T} & \geq h_{T}\left(\xi_{T}\right) \\
x_{1} & \triangleleft \mathcal{F}_{1} \\
& \vdots \\
x_{T} & \triangleleft \mathcal{F}_{T} .
\end{aligned}
$$

Introducing the indicator function

$$
\psi_{\mathbb{X}}(x)= \begin{cases}0 & \text { if } x \in \mathbb{X} \\ \infty & \text { otherwise }\end{cases}
$$

one may identify the cost function $H(x, \xi)$ as

$$
\begin{aligned}
H(x, \xi)= & c_{0} x_{0}+c_{1}\left(x_{1}, \xi_{1}\right)+\cdots+c_{T}\left(x_{T}, \xi_{T}\right)+\psi_{W_{0} x_{0} \geq h_{0}}+\psi_{A_{1} x_{0}+W_{1} x_{1} \geq h_{1}\left(\xi_{1}\right)} \\
& +\cdots+\psi_{A_{T} x_{T-1}+W_{T} x_{T} \geq h_{T}\left(\xi_{T}\right)} .
\end{aligned}
$$

Notice that $\mathbb{E}\left[\psi_{A_{t} x_{t-1}+W_{t} x_{t} \geq h_{t}\left(\xi_{t}\right)}\right]<\infty$ only if $A_{t} x_{t-1}+W_{t} x_{t} \geq h_{t}\left(\xi_{1}\right)$ almost surely.

Lemma 3.5. If the functions $\left(x_{t}, \xi_{t}\right) \mapsto c_{t}\left(x_{t}, \xi_{t}\right)$ as well as the functions $\xi_{t} \mapsto$ $h_{t}\left(\xi_{t}\right)$ are convex, then $(x, \xi) \mapsto H(x, \xi)$ is convex.

Proof. This follows from the fact that $\left\{\left(x_{t}, x_{t-1}, \xi_{t}\right): A_{t} x_{t-1}+W_{t} x_{t} \geq h_{t}\left(\xi_{t}\right)\right\}$ are convex sets.

Example 3.6. If $c(x, \xi)$ is not convex in both variables, but just in $x$ and $\xi$ separately, no relation between the original problem and the expectation-reduced problem holds. As a simple example, take the problem

$$
\min \{\xi \cdot x: \xi \leq x \leq 1\}
$$

with $\xi \sim$ Uniform $[0,1]$. Then

$$
\frac{1}{3}=\mathbb{E} \xi^{2}=\mathbb{E}[\min \{\xi \cdot x: \xi \leq x \leq 1\}]<\min \{\mathbb{E} \xi \cdot x: \mathbb{E} \xi \leq x \leq 1\}=\frac{1}{4} .
$$


On the other hand, for the problem

$$
\min \{-\xi \cdot x:-\xi \leq-x \leq 0\}
$$

we get

$$
-\frac{1}{3}=-\mathbb{E} \xi^{2}=\mathbb{E}[\min \{-\xi \cdot x:-\xi \leq-x \leq 0\}]>\min \{-\mathbb{E} \xi \cdot x:-\mathbb{E} \xi \leq-x \leq 0\}=-\frac{1}{4} .
$$

Notice that $c(\xi)=\xi$ or $c(\xi)=-\xi$ and $h(\xi)=\xi$ or $h(\xi)=-\xi$ are convex in $\xi$, but $(x, \xi) \mapsto \xi \cdot x$ or $(x, \xi) \mapsto-\xi \cdot x$ are not convex in both variables jointly.

4. Upper bounds by inserting (sub)solutions. Solving a real-world stochastic program is often prohibitive from a computational point of view. Many problems have a long planning horizon, which entails serious tractability issues. In such cases, a typical way out is to solve simpler problems by considering simpler stochastic tree models and then fixing the decisions (starting from the root) in the full stochastic program. In these cases it is relevant for decision makers to know how well the solution of a simplified problem performs compared to the corresponding full-size problem. Even in cases where the full stochastic program is not solvable, one may try to solve simpler problems for finding upper bounds and proceed to find tighter bounds, if the gap between the lower and the upper bound is too large.

In this section we revise and extend different procedures to derive upper bounds of the optimal objective function. In general upper bounds are obtained by constraining (some or all) the decision variables to be equal to predetermined fixed values, like the expected value solution, the reference scenario solution, or the optimal first stage decision of a group subproblem. From an algorithmic perspective, these approaches requires us to solve problems with smaller dimension than the original one, which is a computational advantage.

Denote by $v(\mathbb{P}, x)$ the value of the objective; when the decision $x$ is inserted

$$
v(\mathbb{P}, x)=\mathcal{R}_{\mathbb{P}}[H(x, \cdot)] .
$$

Since any decision $x$ cannot be better than the optimal decision, we have

$$
v(\mathbb{P}, x) \geq v^{*}(\mathbb{P}), x \in \mathbb{X} .
$$

Inserting a fixed decision $x_{0: t-1}^{+}$for times $0, \ldots, t-1$ and optimizing only the decisions for times $t, \ldots, T$ is denoted by

$$
v^{*}\left(\mathbb{P}, x_{0: t-1}^{+}\right)=\min \left\{\mathcal{R}_{\mathbb{P}}\left[H\left(\left[x_{0: t-1}^{+}, x_{t: T}\right], \cdot\right)\right]: x_{t: T} \triangleleft \mathfrak{F}_{t: T}\right\} .
$$

The following relation holds true.

THEOREM 4.1.

$$
v\left(\mathbb{P}, x_{0: T-1}^{+}\right) \geq v^{*}\left(\mathbb{P}, x_{0: t-1}^{+}\right) \geq v^{*}(\mathbb{P}) .
$$

Proof. Since the feasible region of $v\left(\mathbb{P}, x_{0: T-1}^{+}\right)$is obviously smaller than the one of $v^{*}\left(\mathbb{P}, x_{0: t-1}^{+}\right), t=0, \ldots, T-2$, which in turn is smaller than the one of $v^{*}(\mathbb{P})$, the relation is obviously satisfied.

Special cases of the concept discussed above are represented by the following class of measures as already introduced in [11] in the linear case. 
Definition 4.2. By denoting $\bar{x}_{0: t-1}$, for times $0, \ldots, t-1$, the optimal solution of the expectation-reduced problem (3.1), the expected result at stage $t$ of the expected value solution, $E E V^{t}(t=1, \ldots, T)$, is defined as

$$
E E V^{t}=v^{*}\left(\mathbb{P}, \bar{x}_{0: t-1}\right)=\min \left\{\mathcal{R}_{\mathbb{P}}\left[H\left(\left[\bar{x}_{0: t-1}, x_{t: T}\right], \cdot\right)\right]: x_{t: T} \triangleleft \mathfrak{F}_{t: T}\right\} .
$$

See [5] for an alternative definition.

Because of relation (4.2), we have

$$
E E V^{t+1} \geq E E V^{t} \geq v^{*}(\mathbb{P}), \quad t=1, \ldots, T-1 .
$$

Similarly, we have the following.

Definition 4.3. $\operatorname{MEVRS}^{t}(t=1, \ldots, T)$, defined as

$$
\operatorname{MEVRS}^{t}=v^{*}\left(\mathbb{P}, x_{0: t-1}^{+\omega_{i}}\right)=\min \left\{\mathcal{R}_{\mathbb{P}}\left[H\left(\left[x_{0: t-1}^{+\omega_{i}}, x_{t: T}\right], \cdot\right)\right]: x_{t: T} \triangleleft \mathfrak{F}_{t: T}\right\},
$$

is obtained by inserting the optimal solution $x_{0: t-1}^{+\omega_{i}}$ until stage $t-1$ of the deterministic problem under a reference scenario $\omega_{i} \in \mathfrak{N}_{T}$.

It is worth pointing out that the problems $E E V^{t}$ and $M E V R S^{t}, t=1, \ldots, T$, could be infeasible since too many variables are fixed to their deterministic solution values. In this case no valid upper bounds can be obtained. However, even when the difference $v^{*}\left(\mathbb{P}, \bar{x}_{0: t-1}\right)-v^{*}(\mathbb{P})$ is large or in the extreme case infinite, the deterministic solution could still carry useful information for the stochastic one. This is evaluated by the multistage expected skeleton solution value $M E S S V^{t}, t=1, \ldots, T$, from [10] and [11] based on the following procedure:

1. fix at zero (or at the lower bound) all the variables which are at zero (or at the lower bound) in the expected value model;

2. solve the related stochastic program.

$M E S S V^{t}$ is defined as follows.

Definition 4.4. By denoting $\bar{x}_{0: t-1}^{0}$ for stages $0, \ldots, t-1$, the optimal solution variables which are at zero or at their lower bound in the expectation-reduced problem (3.1), the multistage expected skeleton solution value at stage $t, M E S S V^{t}$ $(t=1, \ldots, T)$, is given by

$$
M E S S V^{t}=v^{*}\left(\mathbb{P}, \bar{x}_{0: t-1}^{0}\right)=\min \left\{\mathcal{R}_{\mathbb{P}}\left[H\left(\left[\bar{x}_{0: t-1}^{0}, x_{t: T}\right], \cdot\right)\right]: x_{t: T} \triangleleft \mathfrak{F}_{t: T}\right\} .
$$

Again

$$
M E S S V^{t+1} \geq M E S S V^{t}, \quad t=1, \ldots, T-1 .
$$

Such sequences of upper bounds allow us to understand why deterministic solutions do not perform well. Sometimes the deterministic model selects the wrong positive variables and sometimes the choice of out-of-basis variables is fine, but the values are off.

First-stage solutions can also be fixed to values derived from other types of simplified problems, like pair subproblems described before. This is the case of the MEPEV, as introduced in [11] in the linear case.

DEFINITION 4.5. Let $x_{0: 0}^{+i^{(2)}}$ be the optimal first-stage decision to the pair subproblem $v^{*}\left(P_{i}^{(2)}\right), i \neq 1$; then $M E P E V$ is defined as follows:

$$
\begin{aligned}
M E P E V & :=\min _{i \neq 1} v^{*}\left(\mathbb{P}, x_{0: 0}^{+i^{(2)}}\right) \\
& =\min _{i \neq 1} \min \left\{\mathcal{R}_{\mathbb{P}}\left[H\left(\left[x_{0: 0}^{+i^{(2)}}, x_{1: T}\right], \cdot\right)\right]: x_{1: T} \triangleleft \mathfrak{F}_{1: T}\right\} .
\end{aligned}
$$


$M E P E V$ represents the minimum optimal value among those obtained by solving the original stochastic program (1.1), using the optimal first-stage solution of each pair subproblem $v^{*}\left(P_{i}^{(2)}\right), i \neq 1$. As a consequence of (4.2), the following chain of inequalities holds true.

THEOREM 4.6.

$$
v^{*}(\mathbb{P}) \leq M E P E V \leq M E V R S^{1} \leq \cdots \leq M E V R S^{T} .
$$

Proof. See Theorem 4.1 for the proof.

The $M E P E V$ definition can be generalized by considering the optimal first-stage decision to other subproblems $v^{*}\left(P_{i}^{(j)}\right), i \neq 1$, introduced in section 2 .

DeFinition 4.7. Let $x_{0: 0}^{+i^{(j)}}$ be the optimal first-stage decision to the subproblem $v^{*}\left(P_{i}^{(j)}\right)$; then MESEV $V^{j}$ is defined as follows:

$$
\begin{aligned}
\operatorname{MESEV} V^{j} & :=\min _{i} v^{*}\left(\mathbb{P}, x_{0: 0}^{+i^{(j)}}\right) \\
& =\min _{i} \min \left\{\mathcal{R}_{\mathbb{P}}\left[H\left(\left[x_{0: 0}^{+i^{(j)}}, x_{1: T}\right], \cdot\right)\right]: x_{1: T} \triangleleft \mathfrak{F}_{1: T}\right\} .
\end{aligned}
$$

$M E S E V^{j}$ represents the minimum optimal value among those obtained by solving the original stochastic program (1.1), using the optimal first-stage solution of each subproblem $v^{*}\left(P_{i}^{(j)}\right), j \neq i$. For all these subproblems

$$
v^{*}(\mathbb{P}) \leq M E S E V^{j} .
$$

Finally, we study the quality of the upper bound if first-stage decisions are fixed to the values of the first-stage solutions of a smaller subproblem defined below. To this end, we refer to the following well-known theorem.

Theorem 4.8 (Pflug and Pichler [17]). Let $\mathbb{P} \sim(\Omega, \mathfrak{F}, P, \xi)$ be a tree model with filtration $\mathfrak{F}$ and $\tilde{\mathbb{P}} \sim(\tilde{\Omega}, \tilde{\mathfrak{F}}, \tilde{P}, \tilde{\xi})$ be an alternative tree model of the same height with filtration $\tilde{\mathfrak{F}}$. The nested distance between $\mathbb{P}$ and $\tilde{\mathbb{P}}$ is defined as

$$
d(\mathbb{P}, \tilde{\mathbb{P}})=\inf _{\pi} \iint\|\xi(\omega)-\tilde{\xi}(\tilde{\omega})\| \pi(\mathrm{d} \omega, \mathrm{d} \tilde{\omega}),
$$

where $\pi$ is a probability measure with (conditional) marginals $P$ and $\tilde{P}$, i.e.,

$$
\begin{aligned}
& \pi\left(A \times \tilde{\Omega} \mid \mathfrak{F}_{t} \otimes \tilde{\mathfrak{F}}_{t}\right)=P\left(A \mid \mathfrak{F}_{t}\right) \text { and } \\
& \pi\left(\Omega \times B \mid \widetilde{F}_{t} \otimes \tilde{\mathfrak{F}}_{t}\right)=\tilde{P}\left(B \mid \tilde{\mathfrak{F}}_{t}\right), \quad t=0, \ldots T,
\end{aligned}
$$

where $A \in \mathfrak{F}_{T}$ and $B \in \tilde{\mathfrak{F}}_{T}$ and $\mathfrak{F}_{t} \otimes \tilde{\mathfrak{F}}_{t}$ represents the product of sigma algebras. Consider the multistage stochastic optimization problem

$$
v^{*}(\mathbb{P}):=\inf \left\{\mathbb{E}_{P} H(\xi, x): x \triangleleft \mathfrak{F}, x \in \mathbb{X}\right\},
$$

where $\mathbb{X}$ is convex and $L$ is convex in $x$ for any $\xi$ fixed, and Lipschitz with constant $L$ in $\xi$ for any $x$ fixed. Let $\tilde{x}^{*}$ be a solution of

$$
v^{*}(\tilde{\mathbb{P}}):=\inf \left\{\mathbb{E}_{\tilde{P}} H(\xi, x): x \triangleleft \tilde{\mathfrak{F}}, x \in \mathbb{X}\right\},
$$

and let $x^{+}(\xi)=\mathbb{E}_{\pi}\left(\tilde{x}^{*} \mid \xi\right)$, where $\pi$ is the optimizing measure is (4.11). Then $x^{+}$is an approximate solution of $(4.12)$ in the sense that

$$
0 \leq v\left(\mathbb{P}, x^{+}\right)-v^{*}(\mathbb{P}) \leq L \cdot d(\mathbb{P}, \tilde{\mathbb{P}}) .
$$


Proof. See [17] for the proof.

Based on this theorem we may formulate the following corollary.

COROLlary 4.9. Let the assumptions of the previous theorem be fulfilled. Let $\tilde{x}_{0}^{*}$ be the first-stage decision of the simpler problem (4.13). Fixing this solution as the first-stage decision of the original problem (4.12) we get

$$
v^{*}(\mathbb{P}) \leq v\left(\mathbb{P}, \tilde{x}_{0}^{*}\right) \leq v^{*}(\mathbb{P})+L \cdot d(\mathbb{P}, \tilde{\mathbb{P}}) .
$$

Proof. Since the first-stage decisions are always deterministic, we have that $x_{0}^{+}=$ $\tilde{x}_{0}^{*}$, and therefore $v\left(\mathbb{P}, \tilde{x}_{0}^{*}\right)=v\left(\mathbb{P}, x_{0}^{+}\right) \leq v\left(\mathbb{P}, x^{+}\right) \leq v^{*}(\mathbb{P})+L \cdot d(\mathbb{P}, \tilde{\mathbb{P}})$.

5. Case study: A multistage inventory problem. This section presents a simple multistage inventory problem adopted to test the bounds introduced before. The problem can be summarized as follows. Random demands have to be satisfied from an inventory. If the demand exceeds the stock, it will be satisfied by rapid orders from a different source, which come at a higher price. At each time step (stage), orders can be placed, and they will be delivered one period later. The objective is to minimize the expected disutility of the total costs where profits are considered as negative costs. Demands are the only random quantities in the model and all financial quantities are assumed to be already discounted to the present.

We introduce the following notation:

Deterministic parameters:

$b_{t} \quad$ the buying price at time $t=0, \ldots, T-1$

$s_{t} \quad$ the selling price at time $t=1, \ldots, T$

$h_{t}$ the inventory holding costs from time $t$ to time $t+1$

$c_{t}$ the procurement costs for extra stock from another retailer at $t=1, \ldots, T$

$d$ the final value of the inventory

$\zeta_{0}$ the initial stock value

Stochastic scenario process:

$\xi_{t} \quad$ the demand at time $t=1, \ldots, T$ (the random scenario process)

Stochastic decision variables:

$x_{t} \geq 0$ the order sizes at time $t=0, \ldots, T-1$

Derived variables:

$\zeta_{t} \quad$ the stock after all sales are effectuated at $t=1, \ldots, T$

If $\zeta_{t}$ is positive (i.e., $\zeta_{t}=\left[\zeta_{t}\right]_{+}$), then $\left[\zeta_{t}\right]_{+}$is the amount of stock which will be carried to the next time step. If $\zeta_{t}$ is negative (i.e., $\zeta_{t}=-\left[\zeta_{t}\right]_{-}$), then the extra procurement costs of $c_{t}\left[\zeta_{t}\right]_{-}$occur at time $t$ and no stock has to be stored until the next time step. The initial stock $\zeta_{0}$ is fixed at a given value. The final stock is valuated with the value $d\left[\zeta_{T}\right]_{+}$.

The cost function is

$$
\sum_{t=0}^{T-1} b_{t} \cdot x_{t}+\sum_{t=1}^{T} c_{t} \cdot\left[\zeta_{t}\right]_{-}+\sum_{t=0}^{T-1} h_{t} \cdot\left[\zeta_{t}\right]_{+}-\sum_{t=1}^{T} s_{t} \cdot \xi_{t}-d \cdot\left[\zeta_{T}\right]_{+},
$$

and the constraints are

$$
\begin{gathered}
x_{t-1}+\left[\zeta_{t-1}\right]_{+}-\xi_{t}=\zeta_{t}=\left[\zeta_{t}\right]_{+}-\left[\zeta_{t}\right]_{-}, \quad t=1, \ldots, T, \\
{\left[\zeta_{t}\right]_{+} \geq 0, \quad t=1, \ldots, T} \\
{\left[\zeta_{t}\right]_{-} \geq 0, \quad t=1, \ldots, T}
\end{gathered}
$$


The first sum in the cost function (5.1) denotes the expected buying cost, while the second sum represents the procurement costs for extra stock from another retailer, the third term is the inventory holding cost between two consecutive periods, and the last two terms are the profits respectively from selling and for the final value of the inventory. Finally the constraints (5.2), (5.3), and (5.4) define the decision variables of the problem and the relation among them.

The objective function is

$$
\min \left\{\mathbb{E}\left[V\left(\sum_{t=0}^{T-1} b_{t} \cdot x_{t}+\sum_{t=1}^{T} c_{t} \cdot\left[\zeta_{t}\right]_{-}+\sum_{t=0}^{T-1} h_{t} \cdot\left[\zeta_{t}\right]_{+}-\sum_{t=1}^{T} s_{t} \cdot \xi_{t}-d \cdot\left[\zeta_{T}\right]_{+}\right)\right]\right\}
$$

where $V$ is a convex disutility function, which we choose as

$$
V(y)= \begin{cases}y^{1+\delta} & \text { if } y \geq 0 \\ y & \text { if } y<0\end{cases}
$$

for a $\delta>0$.

5.1. Computation of bounds for a multistage inventory problem. This section presents some computational tests on the multistage inventory problem.

For this purpose, we consider the scenario tree structure presented in Figure 5.1, with the demand process shown in Figure 5.2: this is a six-stage tree with 5 branches from the root, 4 from each of the second-stage nodes, 3 from each of the third-stage nodes, 3 from each of the fourth-stage nodes, and 3 from each of the fifth-stage nodes, resulting in $k=5 \times 4 \times 3 \times 3 \times 3=540$ scenarios and 806 nodes. We adopt it as a benchmark to evaluate the cost of optimal solutions obtained using the other reduced scenario trees.

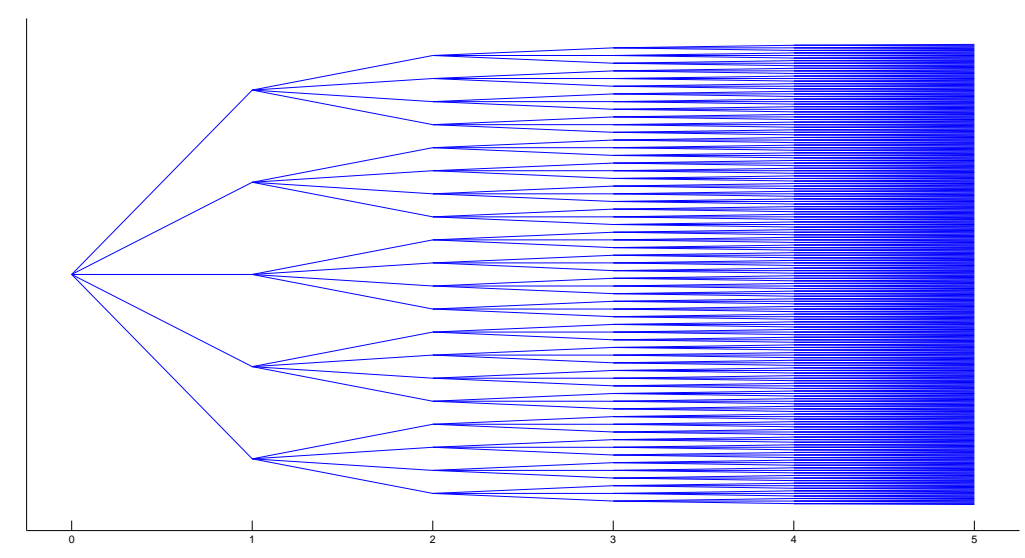

FIG. 5.1. Scenario tree structure adopted for the full multistage stochastic inventory problem with $k=540$ scenarios. 
TABLE 5.1

Buying price $b_{t}$, selling price $s_{t}$, inventory holding cost $h_{t}$ from time $t$ to time $t+1$, and procurement cost $c_{t}$ for extra stock from another retailer at time period $t$.

\begin{tabular}{|c|l|l|l|l|}
\hline$t$ & $b_{t}$ & $s_{t}$ & $h_{t}$ & $c_{t}$ \\
\hline 0 & 3.5 & - & 2 & - \\
1 & 3.6 & 10.7 & 1.9 & 8 \\
2 & 2.3 & 10.5 & 2.1 & 8.1 \\
3 & 2.8 & 10.9 & 2.2 & 7.9 \\
4 & 3 & 10.6 & 2.1 & 7 \\
5 & - & 10 & - & 7.5 \\
\hline
\end{tabular}

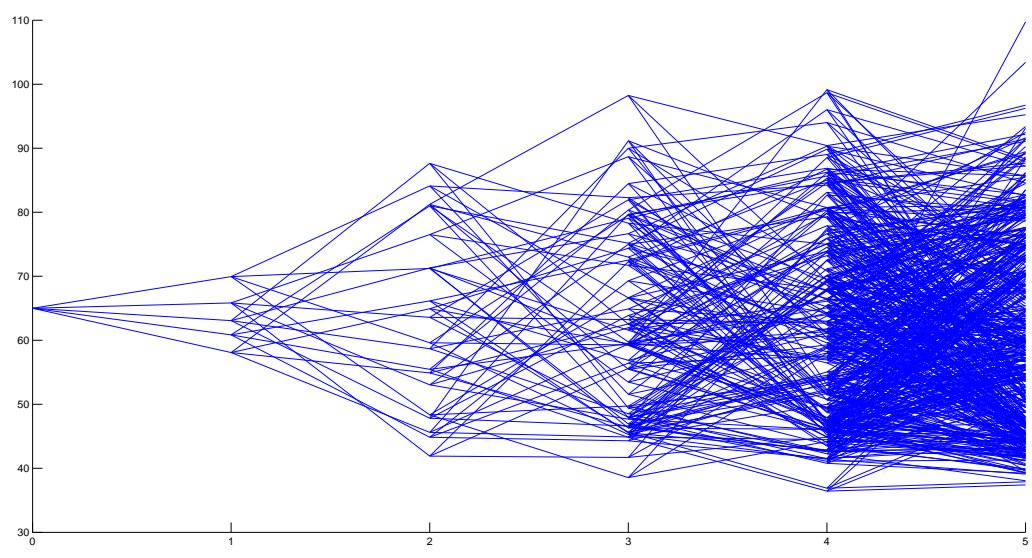

FIG. 5.2. Scenario tree structure with $k=540$ scenarios, generated on the basis of a timeinhomogeneous exponential autoregressive $A R(1)$ model, with demand values $\xi_{t}, t=0, \ldots, 5$, represented as y-values.

The scenario tree has been generated in MATLAB on the basis of a time-inhomogeneous exponential autoregressive $A R(1)$ model for the demand process $\xi_{t}, t=1, \ldots, 6$,

$$
\xi_{t}=e^{c+\varphi \xi_{t-1}+\varepsilon_{t}},
$$

with $c=0, \varphi=\frac{0.8}{\sqrt{t}+1}, \varepsilon_{t}=\frac{1-0.8}{\sqrt{t}+1} \mathcal{N}\left(4,0.3^{2}\right)$ and $\mathcal{N}\left(4,0.3^{2}\right)$ represents a normally distributed random variable with mean parameter $\mu=4$ and standard deviation parameter $\sigma=0.3$.

The value of the process at the root is $\xi_{0}=65$. The final value of the inventory is $d=2$ per unit, the initial stock value is $\zeta_{0}=2 . V(y)$ was chosen as $y^{2}$ and values of buying price $b_{t}$, selling price $s_{t}$, inventory holding cost $h_{t}$, and procurement cost $c_{t}$ at time period $t$ are presented in Table 5.1.

The nonlinear problems derived from our case study have been formulated in AMPL and solved using a MOSEK solver by an interior point algorithm. All the computations have been performed on a 64-bit machine with $12 \mathrm{~GB}$ of RAM and an Intel Core i7-3520M CPU $2.90 \mathrm{GHz}$ processor.

Summary statistics on the size of the optimization problems that AMPL generates are reported in Tables 5.2, 5.3, 5.4, and 5.5 for the six-stage case.

The full stochastic problem is composed by 4304 scalar variables and 2693 constraints. 
TABLE 5.2

Refinement chain of lower bounds made with disjoint subsets for the multistage inventory problem with $k=540$ scenarios.

\begin{tabular}{|l|r|lll|r|}
\hline$j$ & \# subproblems $s$ & & & Objective value & CPU s per subproblem \\
\hline 1 & 540 & $v_{1}^{*}$ & $=$ & -2198.81 & 0.066 \\
5 & 108 & $v_{5}^{*}$ & $=$ & -2193.31 & 0.0705 \\
20 & 27 & $v_{20}^{*}$ & $=$ & -2162.65 & 0.0825 \\
60 & 9 & $v_{60}^{*}$ & $=$ & -2138.17 & 0.108 \\
180 & 3 & $v_{180}^{*}$ & $=$ & -2114.67 & 0.176 \\
540 & 1 & $v^{*}$ & $=$ & -2096.66 & 0.31 \\
\hline
\end{tabular}

TABLE 5.3

Refinement chain of lower bounds obtained by keeping the first scenario fixed in all subsets $\Omega_{i}^{(j)}$ for the multistage inventory problem with $k=540$ scenarios.

\begin{tabular}{|l|l|r|ll|r|}
\hline$j$ & $f$ & \# subproblems $s$ & & Objective value & CPU s per subproblem \\
\hline 2 & 1 & 539 & $v_{2}^{*}$ & $=-2198.77$ & 0.068 \\
8 & 1 & 77 & $v_{8}^{*}$ & $=-2165.61$ & 0.072 \\
12 & 1 & 49 & $v_{12}^{*}=-2157.53$ & 0.077 \\
50 & 1 & 11 & $v_{50}^{*}=-2125.75$ & 0.091 \\
78 & 1 & 7 & $v_{78}^{*}=-2120.92$ & 0.135 \\
540 & 1 & 1 & $v^{*}$ & $=-2096.66$ & 0.31 \\
\hline
\end{tabular}

TABLE 5.4

Refinement chain of lower bounds obtained by keeping the first eight scenarios fixed in all subsets $\Omega_{i}^{(j)}$ for the multistage inventory problem with $k=540$ scenarios.

\begin{tabular}{|l|l|r|ll|r|}
\hline$j$ & $f$ & \# subproblems $s$ & & Objective value & CPU s per subproblem \\
\hline 9 & $1-8$ & 532 & $v_{9}^{*}$ & $=-2198.37$ & 0.065 \\
10 & $1-8$ & 266 & $v_{10}^{*}$ & $=-2189.3$ & 0.071 \\
12 & $1-8$ & 133 & $v_{12}^{*}$ & $=-2176.85$ & 0.073 \\
15 & $1-8$ & 76 & $v_{15}^{*}$ & $=-2165.47$ & 0.079 \\
22 & $1-8$ & 38 & $v_{22}^{*}$ & $=-152.49$ & 0.075 \\
27 & $1-8$ & 28 & $v_{27}^{*}$ & $=-145.58$ & 0.080 \\
36 & $1-8$ & 19 & $v_{36}^{*}$ & $=-2139.86$ & 0.09 \\
46 & $1-8$ & 14 & $v_{46}^{*}$ & $=-2132.18$ & 0.104 \\
84 & $1-8$ & 7 & $v_{84}^{*}$ & $=-2119.82$ & 0.135 \\
141 & $1-8$ & 4 & $v_{141}^{*}=-2114.75$ & 0.195 \\
274 & $1-8$ & 2 & $v_{274}^{*}=-2104.86$ & 0.202 \\
540 & $1-8$ & 1 & $v^{*}$ & $=-2096.66$ & 0.31 \\
\hline
\end{tabular}

We construct refinement chains keeping one or several scenarios fixed in all subsets $\Omega_{i}^{(j)}$ or choosing them disjoint as described in section 2 .

We arbitrarily choose the first $f$ scenarios in the set of available scenarios as fixed for all instances. For any given group size $j$, choosing alternative fixed scenarios can potentially change the sequence of refinements. Knowledge of special structure in a particular instance may lead to better choices of the $f$ fixed scenarios and it is desirable.

Tables 5.3-5.4 provide detailed results obtained respectively by keeping fixed the first and the first eight scenarios in all subsets $\Omega_{i}^{(j)}, i=1, \ldots, s$.

Figure 5.3 refers to the results of Table 5.2 for increasing values of complexity of the calculation measured in CPU seconds. The refinement chain has been constructed choosing disjoint subsets $\Omega_{i}^{(j)}, i=1, \ldots, s$, and following the structure of the scenario tree: 5 branches from the root, 4 from each of the second-stage nodes, 3 from each of 


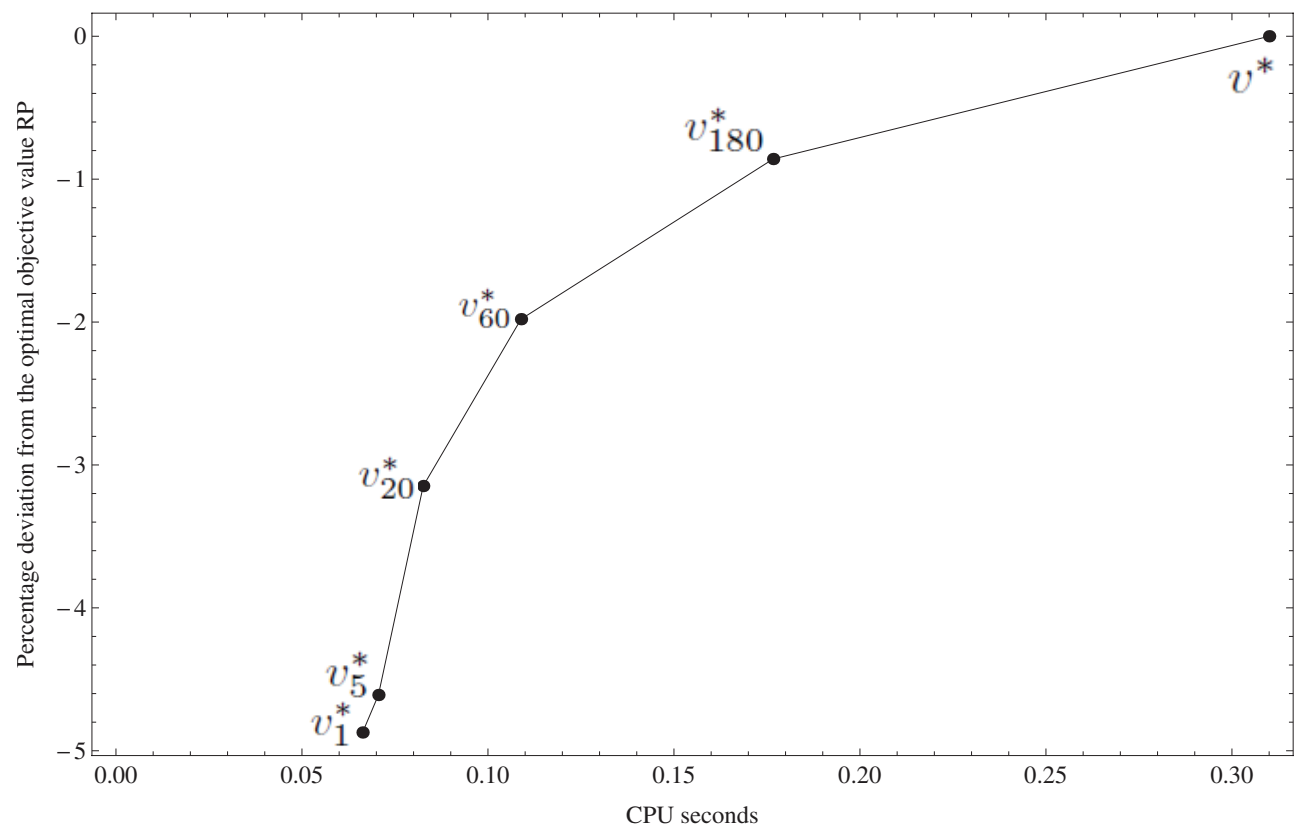

FIG. 5.3. Percentage deviation from the optimal objective value $v^{*}$ of refinement chain reported in Table 5.2 for increasing values of complexity of calculation measured in CPU seconds.

the third-stage nodes, 3 from each of the fourth-stage nodes, and 3 from each of the fifth-stage nodes, resulting in $k=5 \times 4 \times 3 \times 3 \times 3=540$ scenarios.

The first bound $v_{1}^{*}$ in the chain has been obtained by solving all 540 scenario subproblems independently and taking their mean, $v_{5}^{*}$ by considering 180 subproblems made by 5 equidistant scenarios from the five branches in the root, $v_{20}^{*}$ by taking 27 subproblems made by 20 equidistant scenarios from the branches at the second stage, $v_{60}^{*}$ by taking 9 subproblems made by 60 equidistant scenarios from the branches at the third stage, $v_{180}^{*}$ by taking 3 subproblems made by 180 equidistant scenarios from the branches at the fourth stage, and finally the 540 scenarios all together. In this case the refinement chain corresponds to a filtration.

Figures 5.4-5.5 show the percentage deviation of refinement chains reported in Tables 5.3-5.4 for increasing values of complexity of calculation measured in CPU seconds. Figure 5.4 refers to the case of one scenario $(f=1)$ fixed in all subsets $\Omega_{i}^{(j)}$ and Figure 5.5 to the case of eight scenarios $(f=8)$ fixed in all subsets $\Omega_{i}^{(j)}$. The cardinality $j$ of each subset in the chain has been chosen to have the ratio $\frac{540-f}{j-f}=s$ an integer. For instance, with $f=1$ the cardinality of each subproblem in the chain can take only the values $j=2,8,12,50,78,540$, or with $f=8$, $j=9,10,12,15,22,27,36,46,84,141,274,540$.

From the results we observe that $v_{j}^{*}$ improves monotonically with the number of scenarios $j$ of each subproblem (for a fixed number $f$ of fixed scenarios), as proved in (2.8). As expected the worst lower bound in the refinement chains is given by $v_{1}$, which underestimates the optimal value by $4.87 \%$ but requires the lowest CPU time per subproblem (0.066 CPU seconds over 30 runs).

Increasing the group size $j$ in the refinement chain and keeping $f$ fixed significantly 


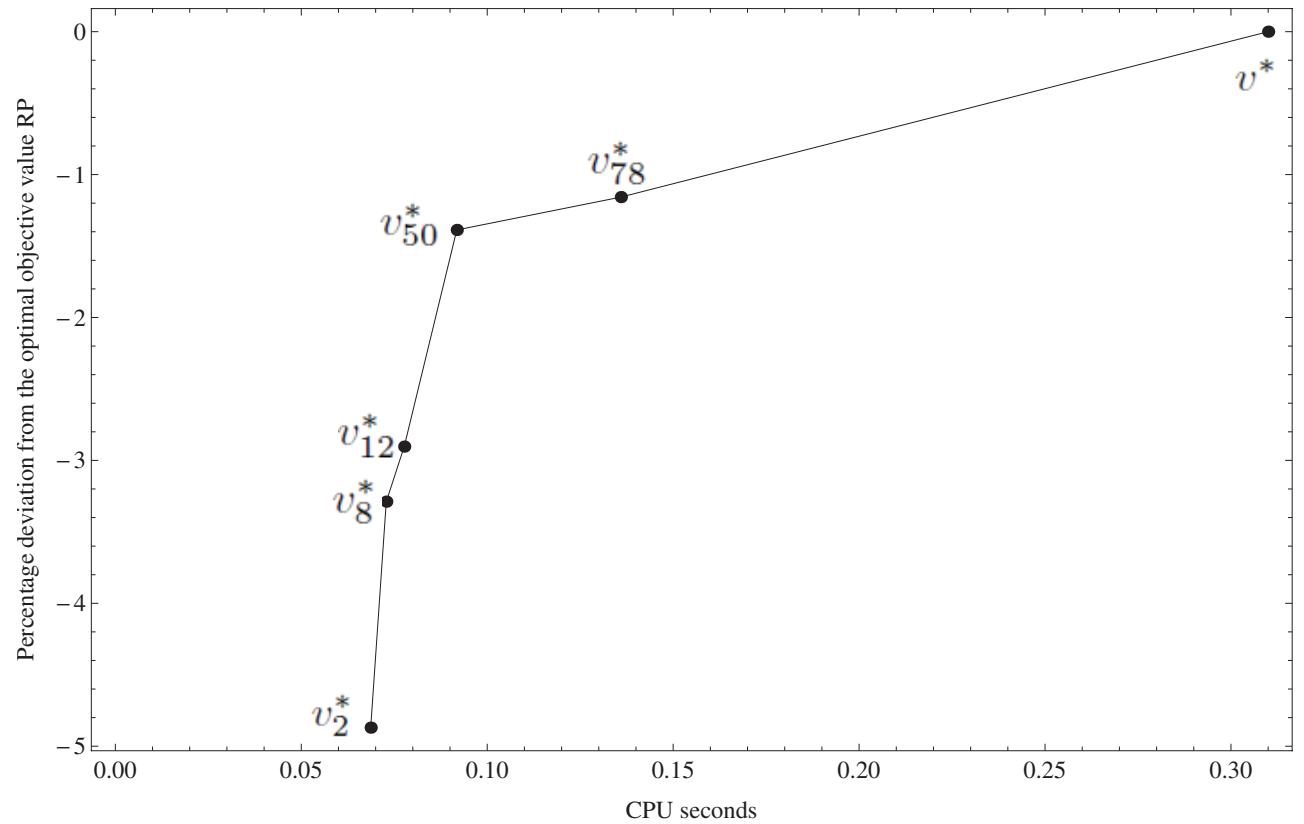

FIG. 5.4. Percentage deviation from the optimal objective value $v^{*}$ of refinement chain reported in Table 5.3 for increasing values of complexity of calculation measured in CPU seconds.

improves the bounds in both construction types, monotonically reaching lower values of percentage deviation. The time required to solve the subproblems in the chain monotonically increases with the dimension of each subproblem reaching the highest value for the biggest scenario tree considered $(j=540)$.

Notice that the same lower bound can be obtained in several ways, keeping fixed a different number of scenarios. This is the case, for example, of lower bound $v_{12}^{*}$ which can be obtained with one fixed scenario and eleven free (see Table 5.3) or with 8 fixed scenarios and 4 free (see Table 5.4). In case of one fixed scenario, $v_{12}^{*}$ is obtained by solving 49 subproblems and it achieves a negative percentage deviation of only 2.9033 . In the case of 8 fixed scenarios, the negative percentage deviation increases to 3.8246 and is obtained by solving a much larger number of subproblems, i.e., 133. Thus we can deduce that better lower bounds are obtained by keeping low the number $f$ of fixed scenarios in all subsets.

Table 5.5 shows the comparison between the stochastic, the expected value solution, and upper bounds by inserting (sub)solutions for the multistage inventory problem, in terms of first-stage decision variables, total cost, and CPU seconds. Inequality (3.2) is verified as well as (4.4), where $E E V^{t}, t=1, \ldots, 5$, is obtained by fixing the stochastic variables until stage $t$ is equal to the expected value solution (see Figure 5.6). Results show that the best upper bound in terms of percentage deviation versus CPU time is given by $E E V^{1}$. The worst upper bound is given by $E E V^{5}$ overestimating the optimal value by $1.69 \%$ but with the lowest CPU time since the dimension of the problem is smaller (0.19 CPU seconds over 30 runs). All EEV ${ }^{t}$ computations take less time compared to the full stochastic problem.

In this example upper bounds defined by formulas (4.8) and (4.10) are sharp, since they correspond exactly to $v^{*}$, but are very expensive in terms of running time 


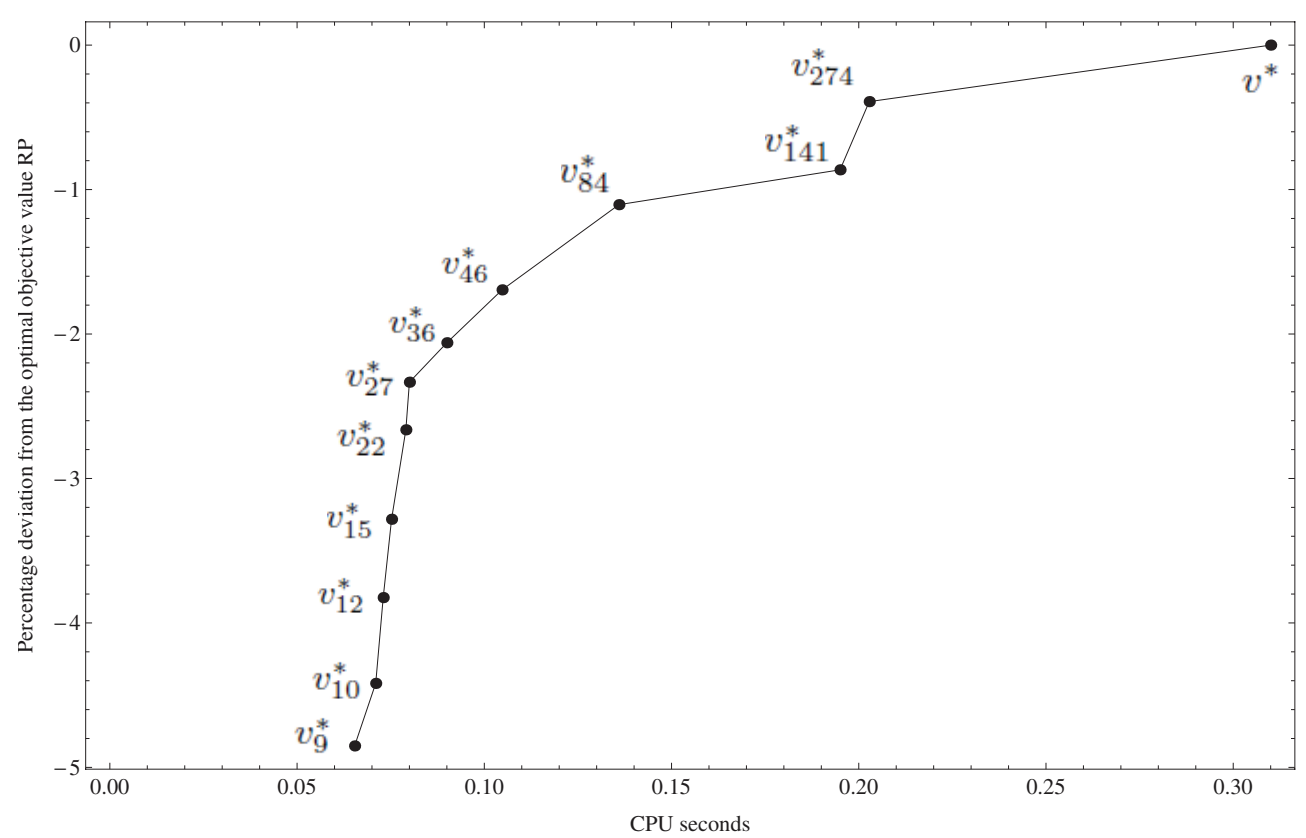

FIG. 5.5. Percentage deviation from the optimal objective value $v^{*}$ of refinement chain reported in Table 5.4 for increasing values of complexity of calculation measured in CPU seconds.

TABLE 5.5

Comparison between the stochastic solution, the expected value solution, and upper bounds by inserting (sub)solutions for the multistage inventory problem ( $k=540$ scenarios).

\begin{tabular}{|l|rrrr|r|r|}
\hline Problem type & $x_{0}$ & {$\left[\zeta_{0}\right]_{+}$} & {$\left[\zeta_{0}\right]_{-}$} & $\zeta_{0}$ & Objective value & CPU s \\
\hline$v^{*}\left(\mathbb{P}_{\mathbb{E}(\xi)}\right)$ & 61.4188 & 2 & 0 & 2 & -2199.70 & 0.02 \\
$v^{*}(\mathbb{P})$ & 63.8405 & 2 & 0 & 2 & -2096.66 & 0.31 \\
$E E V^{1}=v^{*}\left(\mathbb{P}, \bar{x}_{0: 0}\right)$ & 61.4188 & 2 & 0 & 2 & -2095.67 & 0.2486 \\
$E E V^{2}=v^{*}\left(\mathbb{P}, \bar{x}_{0: 1}\right)$ & 61.4188 & 2 & 0 & 2 & -2093.35 & 0.2294 \\
$E E V^{3}=v^{*}\left(\mathbb{P}, \bar{x}_{0: 2}\right)$ & 61.4188 & 2 & 0 & 2 & -2083.58 & 0.2205 \\
$E E V^{4}=v^{*}\left(\mathbb{P}, \bar{x}_{0: 3}\right)$ & 61.4188 & 2 & 0 & 2 & -2071.34 & 0.2122 \\
$E E V^{5}=v^{*}\left(\mathbb{P}, \bar{x}_{0: 4}\right)$ & 61.4188 & 2 & 0 & 2 & -2061.03 & 0.1923 \\
$M E P E V$ & 63.8405 & 2 & 0 & 2 & -2096.66 & 56.69 \\
$M E S E V_{j}$ & 63.8405 & 2 & 0 & 2 & -2096.66 & \\
\hline
\end{tabular}

(56.59 CPU seconds for MEPEV).

$M E P E V=M E S E V_{j}=v^{*}$ means that among all the subproblems considered for the computation of $v_{j}^{*}$, there exists at least one with optimal first-stage solution equal to the optimal first-stage stochastic solution. The high computational cost of such approaches is due to the comparison of the objective function of the full stochastic problem with first-stage solution fixed from each of the subproblems considered. $M E P E V$ should then be considered as an alternative to $E E V^{t}$ upper bounds only in the case that their distance in terms of total cost from $v^{*}$ remains high, which is not the case of the example analyzed here.

Now we consider the case of a problem that is too large to be solved exactly. This is the typical situation in which most modelers would actually resort to the bounding methods described in this paper. 


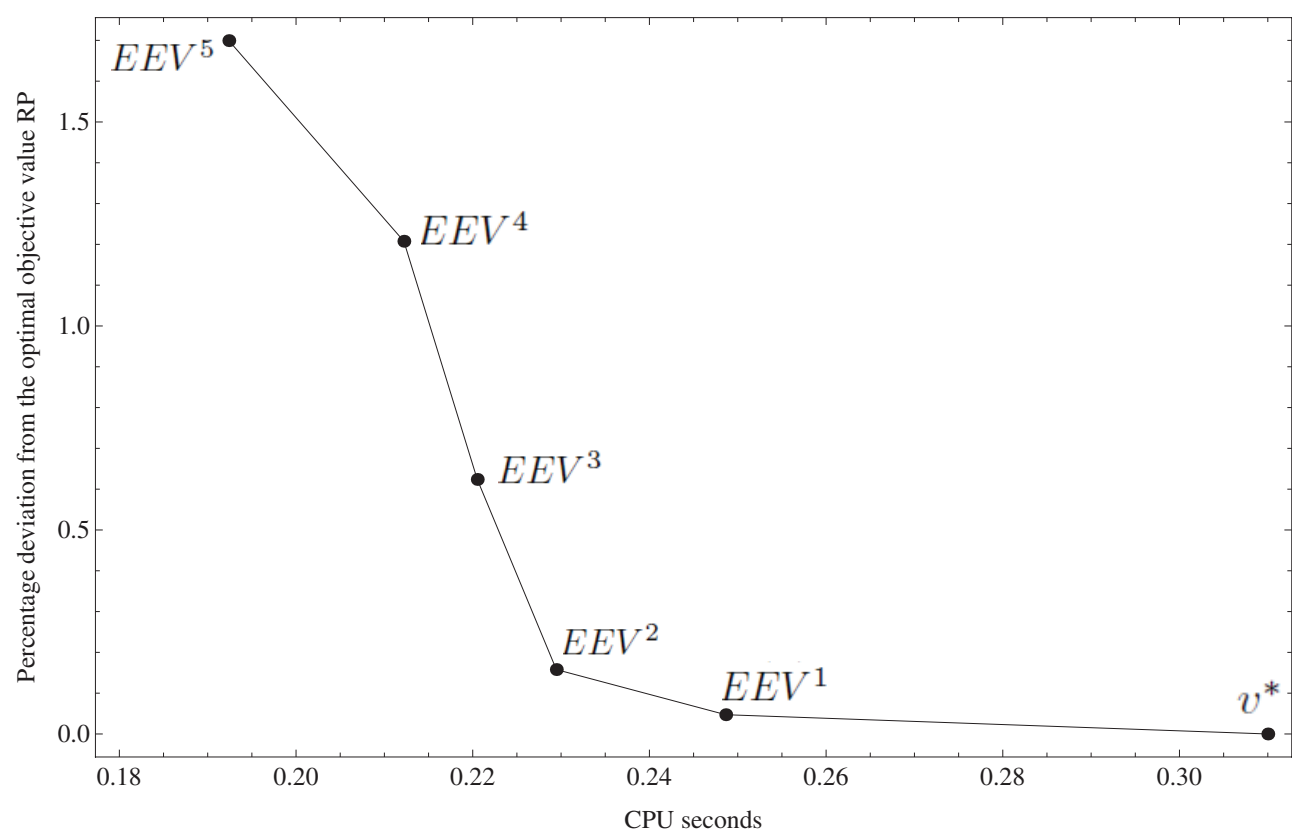

FIG. 5.6. Percentage deviation from the optimal objective value $v^{*}$ of upper bounds $E E V^{t}$, $t=1, \ldots, 5$, reported in Table 5.5 for increasing values of complexity of calculation measured in $C P U$ seconds in the case of a six-stage scenario tree with $k=540$ scenarios.

TABLE 5.6

Buying price $b_{t}$, selling price $s_{t}$, inventory holding cost $h_{t}$ from time $t$ to time $t+1$, and procurement cost $c_{t}$ for extra stock from another retailer at time period $t$ in the case of a scenario tree with 10 stages, 725,760 scenarios, and 1,262,417 nodes.

\begin{tabular}{|l|l|l|l|l|}
\hline$t$ & $b_{t}$ & $s_{t}$ & $h_{t}$ & $c_{t}$ \\
\hline 0 & 3.5 & - & 2 & - \\
1 & 3.6 & 10.7 & 1.9 & 8 \\
2 & 2.3 & 10.5 & 2.1 & 8.1 \\
3 & 2.8 & 10.9 & 2.2 & 7.9 \\
4 & 3 & 10.6 & 2.1 & 7 \\
5 & 3.1 & 10 & 2.3 & 7.5 \\
6 & 3.2 & 10.4 & 1.8 & 7.3 \\
7 & 3.7 & 10.3 & 1.9 & 7.7 \\
8 & 4 & 10.8 & 2.1 & 8.1 \\
9 & - & 10.9 & - & 7.5 \\
\hline
\end{tabular}

For this purpose, we consider a larger scenario tree structure given by a ten-stage tree with 8 branches from the root, 7 from each of the second-stage nodes, 6 from each of the third-stage nodes, 6 from each of the fourth-stage nodes, 5 from each of the fifth-stage nodes, 4 from each of the sixth-stage nodes, 3 from each of the seventhstage nodes, 3 from each of the eight-stage nodes, and 2 from each of the ninth-stage nodes, resulting in $k=8 \times 7 \times 6 \times 6 \times 5 \times 4 \times 3 \times 3 \times 2=725,760$ scenarios and $1,262,417$ nodes. We use it as a benchmark to evaluate the cost of optimal solutions obtained using the other reduced scenario trees.

The scenario tree has been generated as in the previous case with exception of the following values: the value the process at the root was $\xi_{0}=60$, and $\delta$ was set 
TABLE 5.7

Refinement chain of lower bounds made with disjoint subsets for the multistage inventory problem with $k=725,760$ scenarios.

\begin{tabular}{|l|r|ll|r|r|}
\hline$j$ & \# subproblem $s$ & & Objective value & CPU s subprob. & CPU s procedure \\
\hline 8 & 90720 & $v_{8}^{*}$ & $=-3967.61$ & 0.0287 & 2612.29 \\
56 & 12960 & $v_{56}^{*}$ & $=-3941.06$ & 0.0539 & 698.71 \\
336 & 2160 & $v_{336}^{*}$ & $=-3921.15$ & 0.1990 & 430.011 \\
2016 & 360 & $v_{2016}^{*}$ & $=-3902.77$ & 0.9385 & 337.89 \\
10080 & 72 & $v_{10080}^{*}=-3884.23$ & 5.4852 & 394.94 \\
40320 & 18 & $v_{40320}^{*}=-3869.42$ & 27.4512 & 494.123 \\
120960 & 6 & $v_{120960}^{*}=-3857.06$ & 166.5516 & 999.31 \\
362880 & 2 & $v_{362880}^{*}=-3842.61$ & 1274.424 & 2548.848 \\
\hline
\end{tabular}

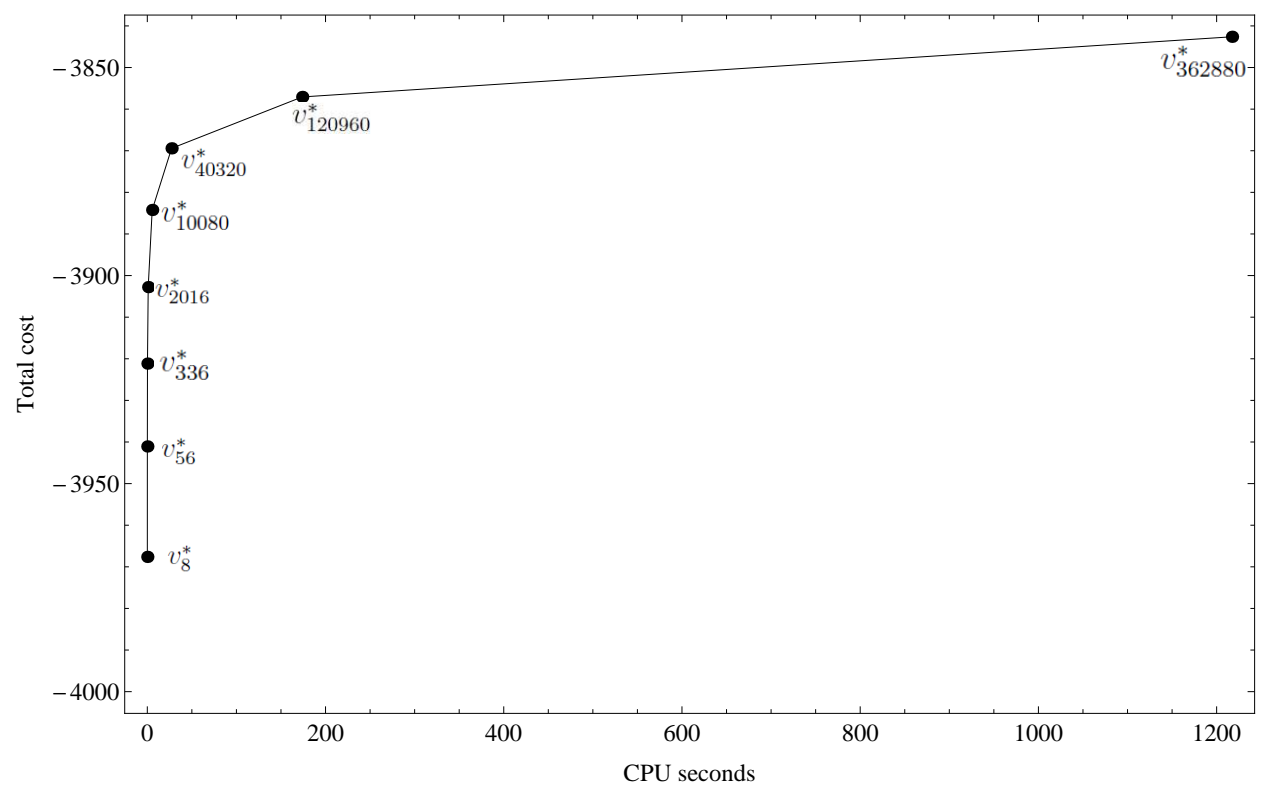

FIG. 5.7. Total costs of refinement chain of lower bounds reported in Table 5.7 for increasing values of complexity of calculation measured in CPU seconds in the case of the 10-stage scenario tree with $k=725,760$ scenarios.

to 3 . The values of buying price $b_{t}$, selling price $s_{t}$, inventory holding cost $h_{t}$, and procurement cost $c_{t}$ at time period $t$ are presented in Table 5.6.

We again use the AMPL environment with the MOSEK solver on a 64-bit machine with $12 \mathrm{~GB}$ of RAM and an Intel Core i7-3520M CPU $2.90 \mathrm{GHz}$ processor.

Summary statistics on the size of the optimization problems that AMPL generates are reported in Table 5.7 and Figure 5.7 for the ten-stage case.

The full stochastic problem is composed of 6,501,188 scalar variables and 3,976,355 constraints.

We construct refinement chains, choosing them disjoint as described in section 2 .

Figure 5.7 refers to the results of Table 5.7 for increasing values of complexity of calculation measured in CPU seconds. The refinement chain has been constructed choosing disjoint subsets $\Omega_{i}^{(j)}, i=1, \ldots, s$, and following the structure of the scenario tree: 8 branches from the root, 7 from each of the second-stage nodes, 6 from each of 
TABLE 5.8

Expected value solution and upper bounds $E E V^{t}, t=8,9$ by inserting (sub)solutions for the multistage inventory problem until stage $t$, in the case of the 10-stage scenario tree with $k=725,760$ scenarios.

\begin{tabular}{|l|r|r|}
\hline Problem type & Objective value & CPU s \\
\hline$v^{*}\left(\mathbb{P}_{\mathbb{E}(\xi)}\right)$ & -3986.71 & 0.09 \\
\hline$E E V^{8}=v^{*}\left(\mathbb{P}, \bar{x}_{0: 8}\right)$ & -3543.16 & 2412.51 \\
$E E V^{9}=v^{*}\left(\mathbb{P}, \bar{x}_{0: 8}\right)$ & -3483.56 & 4254.97 \\
\hline
\end{tabular}

the third-stage nodes, 6 from each of the fourth-stage nodes, 5 from each of the fifthstage nodes, 4 from each of the sixth-stage nodes, 3 from each of the seventh-stage nodes, 3 from each of the eight-stage nodes, and 2 from each of the ninth-stage nodes, resulting in $k=8 \times 7 \times 6 \times 6 \times 5 \times 4 \times 3 \times 3 \times 2=725,760$ scenarios.

The first measure $v_{8}^{*}$ in the chain has been obtained by solving 90,720 scenario subproblems independently and taking the expectation, $v_{56}^{*}$ by considering 12,960 subproblems made by 56 equidistant scenarios from the eight branches in the root, $v_{336}^{*}$ by considering 2160 subproblems made by 336 equidistant scenarios from the branches at the second stage, $v_{2016}^{*}$ by considering 360 subproblems made by 2016 equidistant scenarios from the branches at the third stage, and so on until $v_{362880}^{*}$, obtained by considering 2 subproblems made by 362,880 equidistant scenarios from the branches at the ninth stage. The full problem was unsolvable with the above specified computing environment.

From the results we observe that $v_{j}^{*}$ improves monotonically with the number of scenarios $j$ of each subproblem, as proved in (2.8). As expected the worst lower bound in the refinement chains is given by $v_{8}$ but requires the lowest CPU time per subproblem (0.0287 CPU seconds).

As expected, the bounds improve by increasing the group size $j$ in the refinement chain, with a difference between the first and last bounds in the chain $v_{362880}^{*}-$ $v_{8}^{*}=125\left(=3.25 \% v_{362880}^{*}\right)$. Since the exact value of the full stochastic problem is not available, values of percentage deviations with respect to it cannot be explicitly computed. However, the best calculated lower bound obtained is given by $v_{362880}^{*}$ but with the largest $\mathrm{CPU}$ time per subproblem.

The last column of Table 5.7 reports the CPU seconds for the computation of the bounds sequentially. Evaluating the numerical results from the experimental setting considered, it appears to be more convenient to solve $s=360$ subproblems with $j=2016$ and a time of $337.89 \mathrm{CPU}$ seconds than to calculate the bounds obtained with a lower subproblem cardinality $(j<2016)$, which is also further away from the optimum. Increasing the subproblem cardinality $j$ leads to tighter and tighter bounds as predicted, but at a cost of increasing CPU times.

Another lower bound can be obtained from the expected value problem $v^{*}\left(\mathbb{P}_{\mathbb{E}(\xi)}\right)=$ -3986.71 (see Table 5.8), which is still worse than $v_{8}^{*}$ but solved with a computational time of only $0.09 \mathrm{CPU}$ seconds.

Table 5.8 also shows upper bounds for the multistage inventory problem with $k=725,760$ scenarios in terms of total cost and CPU seconds. The only upper bounds that we were able to calculate were $E E V^{t}, t=8,9$, obtained by fixing all the variables until stage $t$ at the expected value solution, reducing the number of free nodes to $1,088,640$ for $t=8$ and to the number of scenarios for $t=9$, i.e., 725,760. All the other proposed upper bounds $E E V^{t}, t=1, \ldots, 7, M E P E V$, and $M E S E V^{j}$ go out of memory because the number of fixed variables from the expected value solution is not enough to reduce the dimension of the scenario tree to a computationally tractable 
size. The difference between the best upper and lower bounds obtained, $E E V^{8}$ and $v_{362880}^{*}$, respectively, is $299.45\left(7.79 \% v_{362880}^{*}\right)$ and gives information about the range where we should expect to find the total cost of the full stochastic problem that we are not able to compute.

6. Complexity considerations. In the previous section we demonstrated that the calculation of lower bounds requires the solution of many independent problems of smaller size. Since the complexity of a stochastic program grows superlinearly with the number of nodes of the scenario tree, this may result in a considerable reduction of complexity compared to the solution of the full problem, even if the subproblems are all solved sequentially.

To illustrate this, assume that $c(n)$ denotes the worst case execution complexity of a tree structured stochastic program with $n$ nodes, denoted from now on with $\mathcal{P}$. Suppose that the tree has branching factor $b$, i.e., $b=2$ for binary trees, $b=3$ for ternary trees, and so on. For a tree with height $T$, the number of scenarios is $|\Omega|=k=b^{T}$ and the number of nodes is $|\mathfrak{N}|=\left(b^{T+1}-1\right) /(b-1)$. If the subproblems

$$
\Omega_{i}^{(j)}=\left\{\omega_{1}, \ldots, \omega_{f}, \omega_{f+(i-1) \cdot x+1}, \ldots, \omega_{f+i \cdot x}\right\}, \quad i=1, \ldots, s,
$$

are based on $j$ leaf nodes, out of which $f$ are kept fixed, with $x=j-f>0$, one has to solve $s=\left(b^{T}-f\right) /(j-f)$ of them, where each subproblem $\Omega_{i}^{(j)}$ has at most

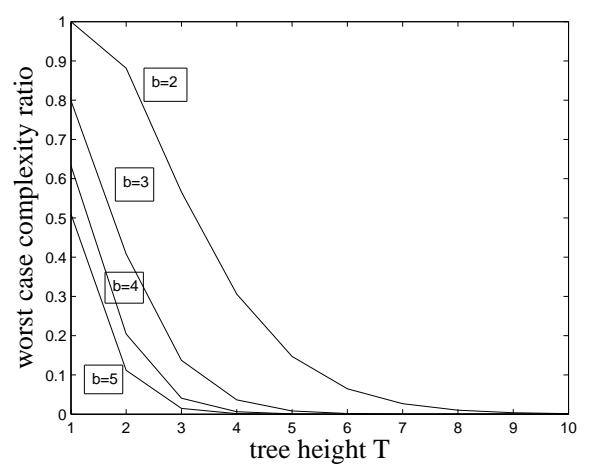

FIG. 6.1. The complexity ratio given by (6.1) using (6.2) for various values of the branching factor $b$ and the tree height $T$. Here the number of leaf nodes for each subproblem is $j=2$ and there are no fixed scenarios among the subproblems, $f=0$.

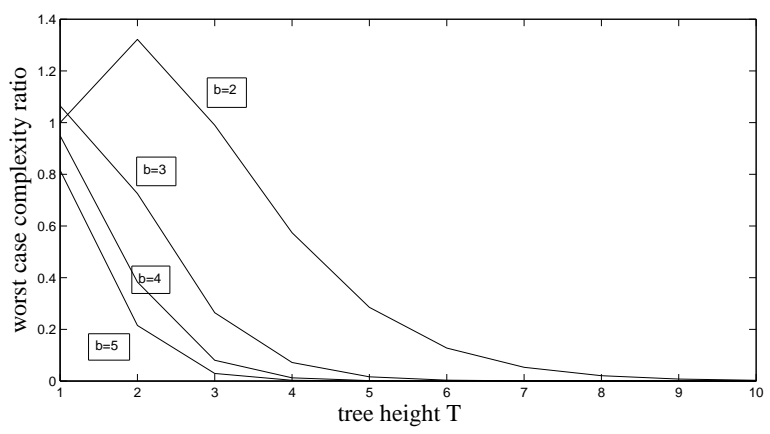

FIG. 6.2. A similar picture as Figure 6.1, but with the choice of 10 leaf nodes for each subproblem, $j=10$, and one fixed scenario $f=1$. 


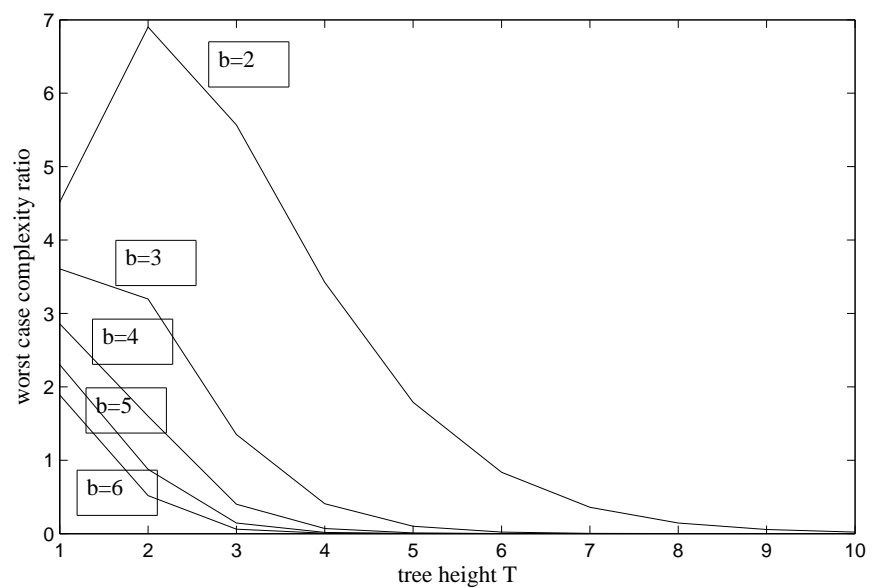

FIG. 6.3. The complexity ratio given by (6.1) using (6.2) for various values of the branching factor $b$ and the tree height $T$. Here the number of leaf nodes for each subproblem is $j=10$ and there are no fixed scenarios among the subproblems, $f=0$.

$\left|\mathfrak{N}_{T}^{(j)}\right|=j T+1$ nodes. Let $\mathcal{P}^{(j)}$ the problem obtained by solving $\Omega^{(j)}=\left\{\Omega_{i}^{(j)} \mid i=\right.$ $1, \ldots, s\}$, with associated lower bound $v_{j}^{*}$. Its complexity is then given by

$$
c\left(\mathcal{P}^{(j)}\right)=c(j T+1) \cdot \frac{b^{T}-f}{j-f}
$$

and the ratio between the worst case complexities of the lower bound calculation $\mathcal{P}^{(j)}$ and the full stochastic problem $\mathcal{P}$ is

$$
\frac{c\left(\mathcal{P}^{(j)}\right)}{c(\mathcal{P})}=\frac{c(j T+1)}{c\left(\left(b^{T+1}-1\right) /(b-1)\right)} \cdot \frac{b^{T}-f}{j-f} .
$$

As an example of a concrete function for $c(\cdot)$ we assume for simplicity that the stochastic program is linear with just one decision variable and one linking constraint per node. If this program is solved by interior point method, then we may use the complexity function

$$
c(n)=O\left(L \cdot n^{3} / \log (n)\right)
$$

given by Anstreicher [1], with $L$ being the data bit size. Inserting (6.2) into (6.1) (with $L=1$ ), one gets the ratio behaviors shown in Figures 6.1, 6.2, and 6.3, which go fast to zero for large $T$, showing the advantage of the proposed procedure. Figures 6.2 and 6.3 exhibit the fact that for small $b$ and $T$, this ratio may even be larger than one.

7. Conclusions. This paper presents strategies to calculate lower and upper bounds for multistage stochastic programs in both the linear and the nonlinear case.

Lower bounds to the optimal solution have been found by relaxation of some constraints and upper bounds by inserting feasible solutions derived by simpler problems.

We introduced lower bounds to the optimal solution by changing the probability measure $P$ (see section 2) or by replacing the scenario process by its expectation (see section 3). In the first case, each lower bound has been calculated by solving group subproblems formed by choosing disjoint scenarios or by keeping some scenarios 
fixed in all subproblems. The monotonicity of the chain of lower bounds in terms of the cardinality of subproblems has been proved as a consequence of the concavity of the probability mapping $P \mapsto v^{*}(P)$ (see Lemma 2.1).

Different upper bounds by inserting (sub)solutions are also analyzed and compared in section 4.

The proposed approach has the important advantage of dividing a given problem into subproblems, the solution of which may easily be parallelized with a good speedup factor due to their independence. Note that lower bounds typically do not lead to feasible solutions of the basic problem, but a small gap between an upper bound (with feasible solution) and a lower bound indicates a nearly optimal solution. Numerical results obtained applying the proposed methodology to a large-scale nonlinear multistage inventory problem have been presented, considering two data sets: one in which the full stochastic problem could be solved and one in which computational difficulties made such solution impossible. Complexity considerations for the computation of the proposed bounds as function of tree depth and branching factor are also discussed.

Acknowledgments. The authors thank the associate editor and the anonymous referees for their helpful comments that improved the quality of the paper.

\section{REFERENCES}

[1] K. M. Anstreicher, Linear programming in $O\left(n^{3} / \ln n \cdot L\right)$ operations, SIAM J. Optim., 9 (1999), pp. 803-812.

[2] J. R. BiRge, The value of the stochastic solution in stochastic linear programs with fixed recourse, Math. Program., 24 (1982), pp. 314-325.

[3] J. R. Birge and F. Louveaux, Introduction to Stochastic Programming, Springer-Verlag, New York, 1997.

[4] D. Dentcheva And A. Ruszczyński, Optimization with stochastic dominance constraints, SIAM J. Optim., 14 (2003), pp. 548-566.

[5] L. F. Escudero, A. Garín, M. Merino, and G. Pérez, The value of the stochastic solution in multistage problems, TOP, 15 (2007), pp. 48-64.

[6] K. Frauendorfer and M. Schürle, Multistage stochastic programming: Barycentric approximation, in Encyclopedia of Optimization, Vol. 3, P. Pardalos, and C. A. Floudas eds., Kluwer Academic, Dordrecht, the Netherlands, 2001, pp. 576-580.

[7] D. Kunn, Generalized Bounds for Convex Multistage Stochastic Programs, Lecture Notes in Economi. and Math. Systems 548, Springer-Verlag, Berlin, 2005.

[8] D. Kunn, Aggregation and discretization in multistage stochastic programming, Math. Program. Ser. A, 113 (2008), pp. 61-94.

[9] D. Kunn, An information-based approximation scheme for stochastic optimization problems in continuous time, Math. Oper. Res., 34 (2009), pp. 428-444.

[10] F. Maggioni and W. S. Wallace, Analyzing the quality of the expected value solution in stochastic programming, Ann. Oper. Res., 200 (2012), pp. 37-54.

[11] F. Maggioni, E. Allevi, and M. Bertocchi, Bounds in multistage linear stochastic programming, J. Optim. Theory Appl., 163 (2014), pp. 200-229.

[12] W. K. Mak, D. P. Morton, And R. K. Wood, Monte Carlo bounding techniques for determining solution quality in stochastic programs, Oper. Res. Lett., 24 (1999), pp. 47-56.

[13] D. P. Morton And R. K. Wood, Restricted-recourse bounds for stochastic linear programming, Oper. Res., 47 (1999), pp. 943-956.

[14] G. Ch. Pflug, A. Ruszczynski, And R. Schultz, On the Glivenko Cantelli problem in stochastic programming: Linear recourse, Math. Oper. Res., 23 (1998), pp. 204-220.

[15] G. Ch. Pflug And W. Römisch, Modeling, Measuring and Managing Risk, World Scientific, Singapore, 2007.

[16] G. Ch. Pflug, Version-independence and nested distributions in multistage stochastic optimization, SIAM J. Optim., 20 (2010), pp. 1406-1420.

[17] G. Ch. Pflug And A. Pichler, A distance for multi-stage stochastic optimization model, SIAM J. Optim., 22 (2012), pp. 1-23.

[18] B. Sandikçi, N. Kong, and A. J. Schaefer, A hierarchy of bounds for stochastic mixedinteger programs, Math. Program. Ser. A, 138 (2012), pp. 253-272. 